\title{
MITO-NUCLEAR SEQUENCING IS PARAMOUNT TO CORRECTLY IDENTIFY SYMPATRIC HYBRIDIZING FISHES
}

\author{
Carla SOUSA-SANTOS ${ }^{1 *}$, Ana M. PEREIRA ${ }^{1}$, Paulo BRANCO ${ }^{2}$, Gonçalo J. COSTA ${ }^{3}$, \\ José M. SANTOS ${ }^{2}$, Maria T. FERREIRA², Cristina S. LIMA ${ }^{1}$, Ignacio DOADRIO ${ }^{4}$, \\ and Joana I. ROBALO ${ }^{1}$ \\ ${ }^{1}$ MARE_Marine and Environmental Sciences Centre, ISPA-Instituto Universitário, Lisboa, Portugal \\ ${ }^{2}$ CEF_Forest Research Centre, Instituto Superior de Agronomia, Universidade de Lisboa, Lisboa, Portugal \\ ${ }^{3}$ Computational Biology and Population Genomics Group $\left(\mathrm{CoBi} \mathrm{G}^{2}\right)$, Centre for Ecology, Evolution and Environmental \\ Changes (CE3C), Faculdade de Ciências, Universidade de Lisboa, Lisboa, Portugal \\ ${ }^{4}$ Department of Biodiversity and Evolutionary Biology, Museo Nacional de Ciencias Naturales, CSIC, Madrid, Spain
}

\begin{abstract}
Sousa-Santos C., Pereira A.M., Branco P., Costa G.J., Santos J.M., Ferreira M.T., Lima C.S., Doadrio I., Robalo J.I. 2018. Mito-nuclear sequencing is paramount to correctly identify sympatric hybridizing fishes. Acta Ichthyol. Piscat. 48 (2): 123-141.
\end{abstract}

Background. Hybridization may drive speciation and erode species, especially when intrageneric sympatric species are involved. Five sympatric Luciobarbus species - Luciobarbus sclateri (Günther, 1868), Luciobarbus comizo (Steindachner, 1864), Luciobarbus microcephalus (Almaça, 1967), Luciobarbus guiraonis (Steindachner, 1866), and Luciobarbus steindachneri (Almaça, 1967) - are commonly identified in field surveys by diagnostic morphological characters. Assuming that i) in loco identification is subjective and observer-dependent, ii) there is previous evidence of interspecific hybridization, and iii) the technical reports usually do not include molecular analyses, our main goal was to assess the concordance between in loco species identification based on phenotypic characters with identifications based on morphometric indices, mtDNA only, and a combination of mito-nuclear markers.

Materials and methods. Specimens of Luciobarbus from six Guadiana River sub-basins were collected and sequenced for the cytochrome $b$ and beta-actin genes. For comparative purposes, samples of Luciobarbus from other 12 river basins were also used. Four levels of taxonomical identification were conducted based on: identification made in the field (in loco identification), cytb gene only, beta-actin gene only, and mito-nuclear combined genomes.

Results. Results showed that interspecific hybridization seems to be high (around 41\%) and likely favoured by non-random mating and the loss of fluvial connectivity. About $34 \%$ of the hybrids showed mito-nuclear discordance. Misidentifications were frequent when only phenotypic characters are considered, and the use of a single mitochondrial gene is not sufficient: the use of two mito-nuclear markers showed that around $82 \%$ of the in loco identifications based on the phenotype were not correct.

Conclusion. Incorrect species assignment likely generated biased results in previous studies on the biology and ecology of Guadiana barbels and in the assignment of conservation status and, consequently, on the establishment of conservation management measures.

Keywords: mito-nuclear incongruence, introgression, hybridization, phenotypic traits, conservation

\section{INTRODUCTION}

Since the 1930s, when the process of natural hybridization began to receive more attention from researchers, two divergent approaches emerged: botanists highlighted its potential for generating diversity (hybrids could occupy new habitats and originate new clades) and zoologists tended to see it as a reproductive mistake that limits diversification and retards evolution (reviewed by Barton 2001, Mallet 2005). According to the latter view, hybridization is the converse of reproductive isolation and challenges the biological species concept, thus the study of hybrids would only be relevant as a tool to understand the development of reproductive isolation (Mallet 2005). 
The emergence of molecular biology techniques exposed the important role of hybridization as a source of genetic variation, a way to generate functional novelty and adaptability, and an important mechanism in the formation of new species (Mallet 2005, Bohling 2016).

According to the literature, the production of viable and fertile hybrids may lead to at least three possible evolutionary scenarios:

If hybridization occurs repeatedly, the extensive gene flow may lead to the extinction of one of the hybridizing species through genetic assimilation (Costedoat et al. 2007 ) or to the merging of the hybridizing species (Taylor et al. 2006)

If hybrids show reduced fitness a hybrid zone may be established, where gene exchange may occur but merging of the parental taxa is prevented (Barton et al. 1985)

If reticulate evolution, when hybrids are at least partially reproductively isolated from the hybridizing species, and the formation of a new, allopolyploid or homoploid, hybrid species occurs (Schumer et al. 2014).

Once formed, fertile F1 hybrids may backcross with one or both the parental species, allowing the occurrence of gene flow and eventually leading to the incorporation of the genes of one species into the genome of the other species (introgressive hybridization) or to the complete merging of the previously isolated hybridizing species ("hybrid swarm") (Scribner et al. 2000). If two hybridizing species are common in their habitats, even low rates of hybridization may have relevant evolutionary consequences. The introgression of genes through hybridization may contribute to adaptive evolution and diversification by enhancing genetic variability (Mallet 2005), but may also raise important concerns regarding species integrity and conservation (Costedoat et al. 2007, Bohling 2016). Evidence for the occurrence of introgression of genes has increased in the last decades, as more molecular techniques became available to the researchers (Arnold 2006). These techniques inclusively detect unsuspicious cases of introgression, namely when parental species and their hybrids are morphologically similar (Gerlach et al. 2016, Paterson et al. 2016).

The high incidence of hybridization in fish taxa seems to be a result of several contributing factors: external fertilization, weak behavioural isolating mechanisms, unequal abundance of the two parental species, competition for limited spawning habitat, decreasing habitat complexity, loss of fluvial connectivity, and susceptibility to secondary contact between recently evolved forms (reviewed by Scribner et al. 2000). The anthropogenic modification of river systems, namely through damming and habitat destruction, may thus be linked with higher levels of hybridization in fish (Hasselman et al. 2014).

Among European cyprinid fish, several cases of hybridization and introgression between native species belonging to the same (Machordom et al. 1990, Congiu et al. 2001, Almodóvar et al. 2008, Lajbner et al. 2009, Geiger et al. 2016) or to different genera (Bianco 1982, Hänfling et al. 2005, Ünver et al. 2005, Pereira et al. 2009, 2014, Aboim et al. 2010, Matondo et al. 2010, Kuparinen et al. 2014, Witkowski et al. 2015) were reported in the last three decades. The hybridization between sympatric species of Luciobarbus inhabiting the Iberian Peninsula was suggested by the finding of morphological intermediates (Almaça 1967, 1972, Machordom et al. 1990, Geiger et al. 2016, Gante et al. 2015) and later corroborated by molecular data (Machordom et al. 1990, Callejas and Ochando 2002, Gante et al. 2015). Recently, a wide study on Iberian Barbus and Luciobarbus pointed to the existence of incomplete reproductive isolation between sympatric species with semi-permeable barriers to gene flow (Gante et al. 2015). This work also showed that, despite the homogenizing effects of hybridization, Iberian barbels might still be discriminated by the combined used of morphological and molecular tools (Gante et al. 2015).

The Guadiana River basin, located westwards from the Gibraltar Strait, harbours five sympatric species of Luciobarbus: Luciobarbus sclateri (Günther, 1868), Luciobarbus comizo (Steindachner, 1864), Luciobarbus microcephalus (Almaça, 1967), Luciobarbus guiraonis (Steindachner, 1866), and Luciobarbus steindachneri (Almaça, 1967) (Fig. 1). Luciobarbus guiraonis is highly abundant in the upper Guadiana (Doadrio et al. 2011) but absent from the lower portion of this river basin, in Portugal. Luciobarbus steindachneri, a Portuguese endemism from the lower Guadiana River, has been taxonomically controversial: several authors considered it a valid species (Kottelat 1997, Bianco and Ketmaier 2001, Almaça and Banarescu 2003, Kottelat and Freyhof 2007) and their work supported its inclusion in FishBase (Froese and Pauly 2017); while other authors consider it an invalid species (Doadrio 1988) or an ecotype of hybrid origin (Gante et al. 2015). Consequently, and although this species was initially described as having a distribution area covering the whole Guadiana River, it was not included in the Spanish checklists of freshwater fishes (Doadrio et al. 1991, 2011, Doadrio 2001, Zamora and Almeida 2015), despite being still present in Portuguese checklists (Cabral et al. 2005).

There are a number of morphometric and meristic measures considered to be diagnostic features of the Luciobarbus species inhabiting the Guadiana River (Almaça 1967, Doadrio et al. 2011) (Fig. 1). Nevertheless, routine biological surveys and technical reports (e.g., for environmental impact studies), and also scientific papers on the biology and ecology of these barbels, rely only on the morphological identification of captured fish using user friendly and in loco traits such as the width of the head, the relative size of the eye, the length of the barbels or the profile of the head (Lobón-Cervia et al. 1984, Encina and Granado-Lorencio 1990, Pires et al. 2001, Morán-López et al. 2005). Conservation efforts are also dependent on an adequate knowledge of barbel populations, and the location of priority areas for protection.

Assuming that i) the use of morphological characters in loco may be subjective and observer-dependent, ii) there is previous evidence of interspecific hybridization between the sympatric Luciobarbus species in the Guadiana River, and iii) technical surveys conducted in 
this river basin (for monitoring the ichthyofauna of the Guadiana National Park or for environmental impact studies, for instance) usually do not include taxonomical identification based on molecular tools, our main goal is to assess the reliability of in loco species identification using morphometric indices, mtDNA only, and a combination of mito-nuclear markers. Considerations about conservation and management studies on Portuguese Luciobarbus and implications for future taxonomical assignments of hybrid species will be drawn.

\section{MATERIALS AND METHODS}

A total of 378 Luciobarbus specimens were collected between 2011 and 2012 in six Guadiana River sub-basins (Fig. 2), using electrofishing (SAMUS725G portable device): Ardila $(n=75)$, Caia $(n=11)$, Chança $(n=66)$, Cobres $(n=35)$, Degebe $(n=33)$, Odeleite $(n=54)$,
Oeiras $(n=39)$, and Vascão $(n=65)$. Fish were identified in the field by two observers, according to practical guidelines followed by the technicians of the local Natural Park "Parque Natural do Vale do Guadiana" for more than two decades, based on the head dorsal profile and on the length of the second pair of barbels relative to the eye (Almaça 1967, Doadrio et al. 2011). Phenotypic traits used to identify Luciobarbus in the Guadiana River are summarized in Table 1.

Juveniles smaller than $10 \mathrm{~cm}(n=72)$ were also sampled but were not identified using the above mentioned diagnostic characters (Table 1) nor used for morphometric analyses, as their identification in the field is considered to be unreliable (Godinho et al. 1997). They were, nevertheless, included in genetic analyses. Dorsal fin clips were taken from all the specimens and preserved in $96 \%$ ethanol as vouchers for the tissue collection of
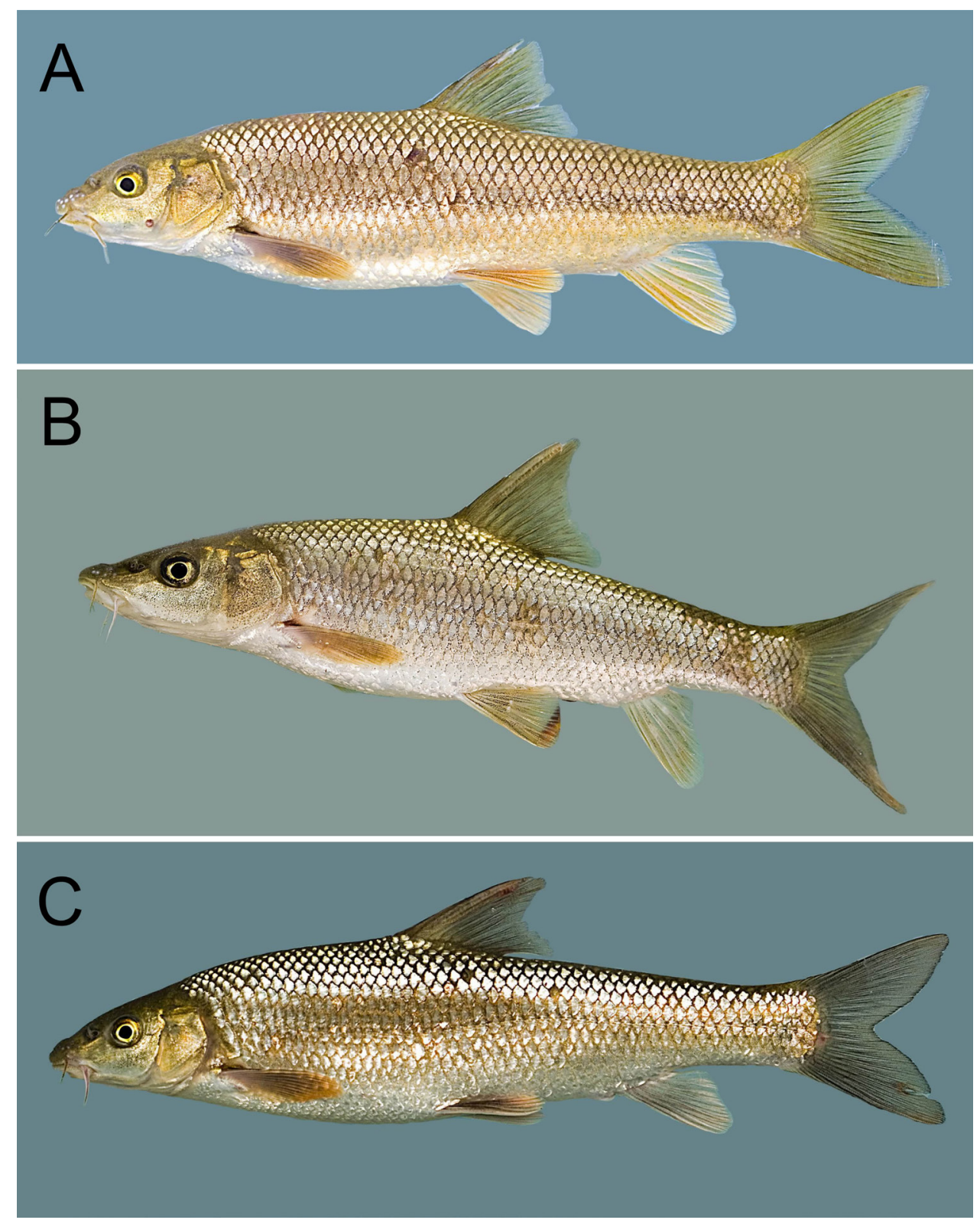

Fig. 1. Sympatric Luciobarbus species in the Portuguese side of the Guadiana River basin: Luciobarbus sclateri (A), Luciobarbus comizo (B), Luciobarbus microcephalus (C) 
Table 1 instructions. One mitochondrial (cytochrome $b$ - cyt $b$ )

Diagnostic phenotypic traits used for the in loco identification of Luciobarbus specimens sampled in the Guadiana River

\begin{tabular}{|c|c|}
\hline Species & Phenotypic trait \\
\hline L. sclateri & $\begin{array}{l}\text { Barbels extending beyond the posterior edge } \\
\text { of the eye }\end{array}$ \\
\hline L. steindachneri & Barbels reaching the middle of the eye \\
\hline L. microcephalus & $\begin{array}{l}\text { Short barbels (not reaching the anterior edge } \\
\text { of the eye) and short head with concave } \\
\text { dorsal profile }\end{array}$ \\
\hline L. comizo & $\begin{array}{l}\text { Short barbels (not reaching the anterior } \\
\text { edge of the eye) and long head with concave } \\
\text { dorsal profile and duck-like snout }\end{array}$ \\
\hline Hybrids & Intermediate characteristics from the above \\
\hline
\end{tabular}

MARE-ISPA (Lisbon, Portugal). Fish were photographed and immediately released to the water.

For comparative purposes and to allow for the validation of nuclear-specific tags (see below), 157 samples of additional Luciobarbus populations (Fig. 2), available from the same tissue collection, were used for genetic analyses: $20 \mathrm{~L}$. comizo from the Tagus River, 26 L. sclateri from rivers Seixe and Arade, and 111 Luciobarbus bocagei (Steindachner, 1864) from nine river basins located throughout the species distribution area (Table 2 and Fig. 2). These samples, except for the Tagus population (where L. bocagei is sympatric with L. comizo), were from populations where only one Luciobarbus species occurs, avoiding the potential noise of current interspecific hybridizations on the nuclear signal. The complete dataset of samples is presented in Table 2.

Molecular data analyses. Total genomic DNA was extracted from fin clips using REDExtract-N-Amp Tissue PCR kits (Sigma-Aldrich) following the manufacturer's and one nuclear gene (beta-actin) were amplified using the primers LCB1new-ACTTGAAGAACCACCGTTG (newly designed, based on the LCB1 primer described by Brito et al. 1997) and HA-CAACGATCTCCGGTTTACAAGAC (Schmidt and Gold 1993) for cyt $b$, and BACTFOR-ATGGATGATGAAATTGCCGC and BACTREV-AGGATCTTCATGAGGTAGTC (Robalo et al. 2007) for beta-actin. PCR conditions followed the ones described in Sousa-Santos et al. (2014). Primers LCB1new and BACTFOR were used for forward sequencing reactions and the PCR products were purified and sequenced at the GATC company (Germany). Obtained sequences were aligned and manually edited using CodonCode Aligner v4.0.4 (CodonCode Corp., USA). All beta-actin and some cyt $b(n=60)$ sequences were newly obtained for this work whilst the remaining cyt $b$ sequences $(n=$ 194) were previously obtained by the research team under the scope of the FISHATLAS project and were already available in GenBank (Table 3). GenBank accession number of all cyt $b$ sequences are presented in Table 3 . The obtained beta-actin sequences were not genotyped (and, thus, not deposited in GenBank) since it was our goal to detect specific tags among the superimposed double peaks (see below). Chromatograms are available to be sent by the authors upon request.

As Luciobarbus species are tetraploid (Ráb and Collares-Pereira 1995), the amplification of the nuclear beta-actin gene generates mixed PCR products and the consequent production of traces with multiple peaks for the majority of the loci. Indeed, if the four alleles exhibit the same nucleotide in a particular locus, a single peak will be read by the sequencer. Contrastingly, it is possible that four nucleotides, one from each allele, may appear superimposed at a given locus of the sequenced gene fragment. Several manual (Sousa-Santos et al. 2005)

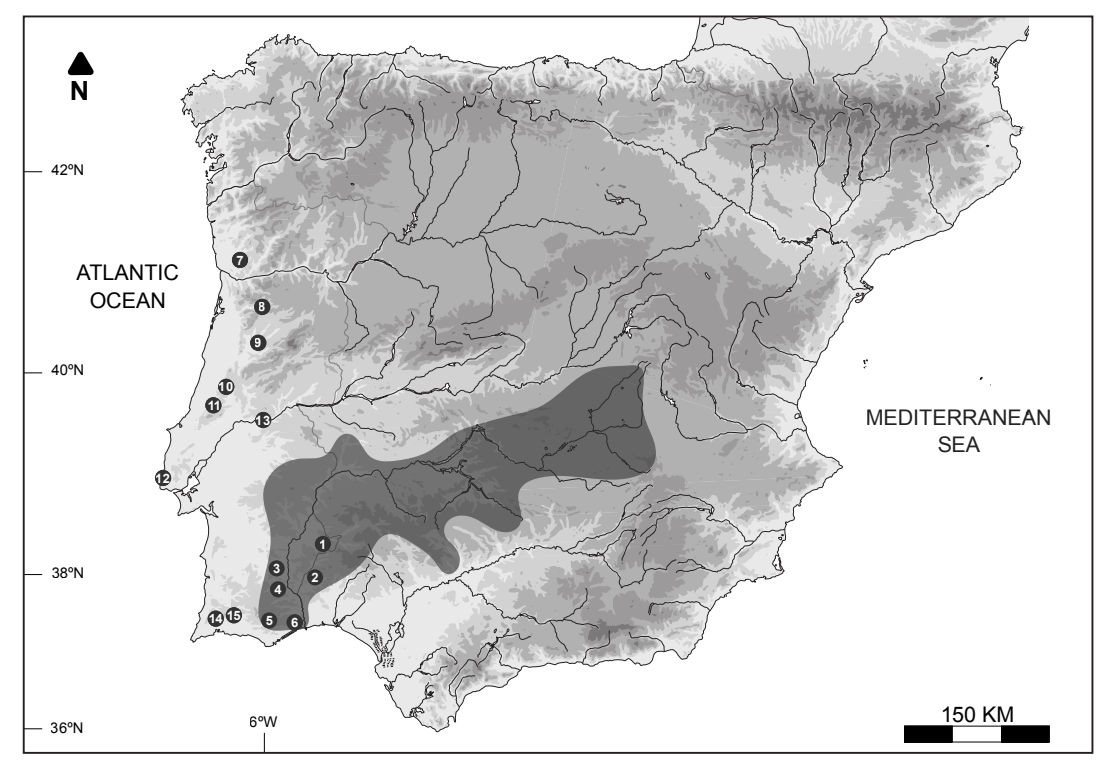

Fig. 2. Sampling locations of Luciobarbus species in the Guadiana River basin (grey shaded): $(1=$ Caia, $2=$ Degebe, $3=$ Ardila, $4=$ Chança, $5=$ Cobres, $6=$ Oeiras, $7=$ Vascão, $8=$ Odeleite $)$ and other rivers $(7=$ Ave, $8=$ Vouga, $9=$ Mondego, $10=$ Lis, $11=$ Alcoa, $12=$ Colares, $13=$ Tagus, $14=$ Seixe, $15=$ Arade) 
Table 2 beta-actin gene to validate the direct sequencing of the

Luciobarbus specimens sampled in the Guadiana River sub-basins $(n=378)$ and in nine river basins located outside the study area $(n=157)$

\begin{tabular}{|c|c|c|c|c|c|c|c|c|}
\hline & & & nber & $\begin{array}{l}f \text { fis } \\
\text { ide }\end{array}$ & $\begin{array}{l}\text { spe } \\
\text { ifica }\end{array}$ & $\begin{array}{l}\text { men } \\
\text { on) }\end{array}$ & (in $l$ & \\
\hline \multirow{9}{*}{ 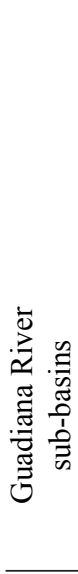 } & River Basin & 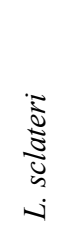 & 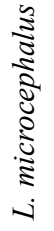 & 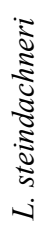 & 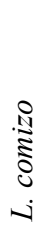 & 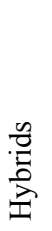 & 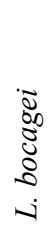 & $\begin{array}{l}\overrightarrow{0} \\
\stackrel{0}{0} \\
0 \\
.00 \\
0 \\
0\end{array}$ \\
\hline & Caia & & & & & & & 11 \\
\hline & Degebe & & & & & & & 33 \\
\hline & Ardila & 11 & 10 & 13 & 11 & 4 & & 26 \\
\hline & Chança & 39 & 12 & 14 & 1 & & & \\
\hline & Cobres & 2 & & & 32 & & & 1 \\
\hline & Oeiras & 9 & 11 & 15 & 3 & & & 1 \\
\hline & Vascão & 34 & 13 & 3 & 15 & & & \\
\hline & Odeleite & 26 & & 26 & 2 & & & \\
\hline \multirow{10}{*}{ 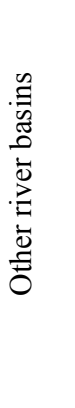 } & Ave & & & & & \multicolumn{3}{|c|}{7} \\
\hline & Vouga & & & & & \multicolumn{3}{|c|}{29} \\
\hline & Mondego & & & & & \multicolumn{3}{|c|}{37} \\
\hline & Lis & & & & & \multicolumn{3}{|c|}{3} \\
\hline & Alcoa & & & & & \multicolumn{3}{|c|}{2} \\
\hline & Colares & & & & & \multicolumn{3}{|c|}{2} \\
\hline & Tagus & & & & 20 & \multicolumn{3}{|c|}{31} \\
\hline & Seixe & 19 & & & & & & \\
\hline & Arade & 7 & & & & & & \\
\hline & Total & 147 & 46 & 71 & 84 & 4 & 111 & 72 \\
\hline
\end{tabular}

In the river basins where more than one Luciobarbus species occur (Tagus and Guadiana), the field identification of the species was made according to the criteria described in the Materials and methods section. As occurred in the Guadiana, the L. comizo specimens from the Tagus were distinguishable from the sympatric $L$. bocagei due to the presence of short barbels (not reaching the anterior edge of the eye) and long head with concave dorsal profile and duck-like snout.

and automated/statistical approaches (Stephens et al. 2001, 2003, Bhangale et al. 2006, Scheet and Stephens 2006, Chen et al. 2007, Dmitriev and Rakitov 2008) have been developed in the past decade to disentangle both gene complements of diploid heterozygotes and hybrids. In some cases, these approaches benefit from the presence of diagnostic heterozygous insertions-deletions mutations (indels) which result in a phase shift in the trace, from the mutation point onwards (Sousa-Santos et al. 2005). For tetraploid individuals which are heterozygous for a given nuclear gene, however, the haplotype determination is not an easy task, especially if indels are present.

Iberian barbels exhibit four copies of the betaactin gene, identical two by two, with several indels in conserved regions (Sousa-Santos unpublished data). These haplotypes may be recovered by using paralog-specific primers, as already described for the direct sequencing of the S7 and growth hormone genes of Barbus and Luciobarbus (see Gante et al. 2011). However, in the presently reported study, as our goal was to use the nuclear mitochondrial cyt $b$ gene (or, on the contrary, to refute the mtDNA-based classification when hybridization signals are detected), we opted for a less expensive and expedite methodology which may be easily replicated in future studies. Thus, traces obtained from the beta-actin gene sequencing of Luciobarbus from the Guadiana River were aligned with CodonCode Aligner v4.0.4 (CodonCode Corp., USA). Automated base calling using the nucleotide ambiguity code (IUPAC) was made by CodonCode Aligner ("calling secondary peaks" function) and each locus was posteriorly manually inspected to search for point mutations which can be used as diagnostic of the species sampled. Samples of L. bocagei, L. sclateri, and L. comizo from other river basins (Table 2) were also manually inspected for support to the former identification of diagnostic loci. Tags identified for L. comizo and L. sclateri from Guadiana specimens were validated with the sequencing results obtained for individuals of the same species from other river basins (respectively, Tagus and Arade/Seixe). Identical procedure was not possible to conduct for the validation of $L$. microcephalus and L. steindachneri tags since these species only occurs in the Guadiana River.

After the identification of the diagnostic loci for the beta-actin gene, each individual was assigned to one of four categories: L. sclateri, L. microcephalus, L. comizo, and hybrid (when the beta-actin sequence shows tags which are specific of two or more Luciobarbus species).

Finally, we built a matrix summarizing the four levels of taxonomical identification for all sampled individuals: identification made in the field (in loco identification) (four categories, corresponding to the four Luciobarbus species), cyt $b$ genome (three categories, corresponding to L. sclateri, L. comizo and L. microcephalus), beta-actin genome (four categories, above mentioned); and mitonuclear combined genome (four categories: L. sclateri, L. microcephalus, L. comizo, and hybrid).

Morphometric analyses. For each of the 276 collected adult specimens, individual images were taken with a PENTAX Optio E85 camera (available to be sent by the authors upon request). Each image was processed in Adobe Photoshop CS5 Extended with an X-Ray Filter to enhance general contrast, adjust brightness and contrast, cropped to reduce file size, and saved as a .tiff file with a traceable unknown ID label (Spec_nnn). Afterwards, each image was analysed using FIJI ImageJ v. 1.49 (Schindelin et al. 2012) with a multipoint tool to assign each point to up to 15 morphometric landmarks (Fig. 3). Image calibration was not possible in the field but instead, XY pixel coordinates were obtained for each point. The resulting matrix was transformed to .tps format and an EDMA (all distances between landmarks) matrix was calculated in PAST v. 2.16 (Hammer et al. 2001), from which 19 morphometric measures adapted from Armbruster (2012) were extracted (Table 4). These measures were selected since they were related with the main features used for the identification of Luciobarbus species, namely the morphology of the head and the relative 
Table 3

Identification of the individuals sampled in the lower Guadiana River sub-basins $(n=194)$, based on different criteria

\begin{tabular}{|c|c|c|c|c|c|c|c|}
\hline \multirow{2}{*}{ Individual ID } & \multirow{2}{*}{ Sub-basin } & \multicolumn{5}{|c|}{ Identification criterion } & \multirow{2}{*}{$\begin{array}{c}\text { GenBank } \\
\text { Accession No. }\end{array}$} \\
\hline & & Field ID & Cytb ID & Beta-actin ID & Mito-nuclear ID & Morphometry & \\
\hline BCG10 & Oeiras & $\mathrm{LC}$ & $\mathrm{LS}$ & LS & LS & & KU368468 \\
\hline BCG11 & Oeiras & $\mathrm{LC}$ & LS & $\mathrm{LC}$ & HYB & & KU368469 \\
\hline BCG8 & Oeiras & $\mathrm{LC}$ & LS & $\mathrm{LC}$ & HYB & & KU368474 \\
\hline BCGAR10 & Ardila & $\mathrm{LC}$ & $\mathrm{LC}$ & $\mathrm{LC}$ & $\mathrm{LC}$ & + & KU368222 \\
\hline BCGAR11 & Ardila & $\mathrm{LC}$ & LS & $\mathrm{LC}$ & HYB & + & KU368383 \\
\hline BCGAR12 & Ardila & $\mathrm{LC}$ & $\mathrm{LC}$ & $\mathrm{LC}$ & $\mathrm{LC}$ & + & KU368223 \\
\hline BCGAR6 & Ardila & $\mathrm{LC}$ & $\mathrm{LC}$ & $\mathrm{LC}$ & $\mathrm{LC}$ & + & KU368228 \\
\hline BCGAR7 & Ardila & $\mathrm{LC}$ & $\mathrm{LC}$ & $\mathrm{LC}$ & $\mathrm{LC}$ & + & KU368229 \\
\hline BCGAR8 & Ardila & $\mathrm{LC}$ & $\mathrm{LC}$ & $\mathrm{LC}$ & $\mathrm{LC}$ & + & KU368230 \\
\hline BCGAR9 & Ardila & $\mathrm{LC}$ & $\mathrm{LC}$ & $\mathrm{LC}$ & $\mathrm{LC}$ & + & KY930962 \\
\hline BCGCO1 & Cobres & $\mathrm{LC}$ & $\mathrm{LC}$ & LS & HYB & + & KU368279 \\
\hline BCGCO10 & Cobres & $\mathrm{LC}$ & $\mathrm{LC}$ & $\mathrm{LC}$ & $\mathrm{LC}$ & & KU368280 \\
\hline BCGCO11 & Cobres & $\mathrm{LC}$ & $\mathrm{LC}$ & $\mathrm{LC}$ & $\mathrm{LC}$ & + & KU368281 \\
\hline BCGCO13 & Cobres & $\mathrm{LC}$ & $\mathrm{LC}$ & HYB & HYB & + & KU368282 \\
\hline BCGCO16 & Cobres & $\mathrm{LC}$ & $\mathrm{LC}$ & $\mathrm{LC}$ & $\mathrm{LC}$ & + & KU368285 \\
\hline BCGCO17 & Cobres & $\mathrm{LC}$ & $\mathrm{LC}$ & $\mathrm{LC}$ & $\mathrm{LC}$ & + & KU368286 \\
\hline BCGCO18 & Cobres & $\mathrm{LC}$ & $\mathrm{LC}$ & $\mathrm{LC}$ & $\mathrm{LC}$ & + & KU368287 \\
\hline BCGCO19 & Cobres & $\mathrm{LC}$ & $\mathrm{LC}$ & $\mathrm{LC}$ & $\mathrm{LC}$ & + & KU368288 \\
\hline BCGCO2 & Cobres & $\mathrm{LC}$ & LS & HYB & HYB & + & KY930963 \\
\hline BCGCO3 & Cobres & $\mathrm{LC}$ & $\mathrm{LC}$ & HYB & HYB & + & KU368290 \\
\hline BCGCO4 & Cobres & $\mathrm{LC}$ & $\mathrm{LC}$ & $\mathrm{LC}$ & $\mathrm{LC}$ & + & KU368291 \\
\hline BCGCO5 & Cobres & $\mathrm{LC}$ & $\mathrm{LC}$ & HYB & HYB & + & KU368292 \\
\hline BCGCO6 & Cobres & $\mathrm{LC}$ & $\mathrm{LC}$ & $\mathrm{LC}$ & $\mathrm{LC}$ & + & KU368293 \\
\hline BCGCO7 & Cobres & $\mathrm{LC}$ & $\mathrm{LC}$ & $\mathrm{LC}$ & $\mathrm{LC}$ & + & KU368294 \\
\hline BCGCO8 & Cobres & $\mathrm{LC}$ & $\mathrm{LC}$ & $\mathrm{LC}$ & $\mathrm{LC}$ & + & KU368295 \\
\hline BCGCO9 & Cobres & $\mathrm{LC}$ & $\mathrm{LC}$ & HYB & HYB & + & KU368296 \\
\hline BCGDE1 & Cobres & $\mathrm{LC}$ & LS & HYB & HYB & + & KY930964 \\
\hline BCGO2 & Cobres & $\mathrm{LC}$ & $\mathrm{LC}$ & HYB & HYB & + & KY930965 \\
\hline BCGO3 & Cobres & $\mathrm{LC}$ & LS & LS & LS & + & KU368476 \\
\hline BCGO5 & Cobres & $\mathrm{LC}$ & LS & LS & LS & + & KY930966 \\
\hline BCGO6 & Cobres & $\mathrm{LC}$ & $\mathrm{LC}$ & HYB & HYB & + & KY930967 \\
\hline BCGOD1 & Odeleite & $\mathrm{LC}$ & $\mathrm{LC}$ & $\mathrm{LC}$ & $\mathrm{LC}$ & + & KY930968 \\
\hline BCGOD2 & Odeleite & $\mathrm{LC}$ & LS & LS & LS & & KU368443 \\
\hline BCGV1 & Vascão & $\mathrm{LC}$ & $\mathrm{LC}$ & $\mathrm{LC}$ & $\mathrm{LC}$ & & KU368320 \\
\hline BCGV10 & Vascão & $\mathrm{LC}$ & $\mathrm{LC}$ & HYB & HYB & + & KY930969 \\
\hline BCGV2 & Vascão & $\mathrm{LC}$ & LS & LS & LS & + & KU368490 \\
\hline BCGV3 & Vascão & $\mathrm{LC}$ & $\mathrm{LC}$ & LS & HYB & + & KU368318 \\
\hline BCGV4 & Vascão & $\mathrm{LC}$ & $\mathrm{LC}$ & $\mathrm{LC}$ & $\mathrm{LC}$ & + & KU368317 \\
\hline BCGV5 & Vascão & $\mathrm{LC}$ & $\mathrm{LC}$ & HYB & HYB & + & KU368316 \\
\hline BCGV6 b & Vascão & $\mathrm{LC}$ & $\mathrm{LC}$ & HYB & HYB & + & KU368319 \\
\hline BCGV8 & Vascão & $\mathrm{LC}$ & $\mathrm{LC}$ & HYB & HYB & + & KU368315 \\
\hline BCGV9 & Vascão & $\mathrm{LC}$ & $\mathrm{LC}$ & LS & HYB & & KU368321 \\
\hline BMG15 & Oeiras & LM & LM & LM & LM & & KY930970 \\
\hline BMG17 & Oeiras & LM & LM & LM & LM & & KY930971 \\
\hline BMG3 & Oeiras & LM & LM & LM & LM & & KY930972 \\
\hline BMG4 & Oeiras & LM & LM & HYB & HYB & & KY930973 \\
\hline BMG5 & Oeiras & LM & LM & LM & LM & & KY930974 \\
\hline BMG6 & Oeiras & LM & LS & HYB & HYB & & KU368477 \\
\hline BMG8 & Oeiras & LM & LM & HYB & HYB & & KY930975 \\
\hline BMGAR1 & Ardila & LM & $\mathrm{LC}$ & LS & HYB & + & KU368242 \\
\hline BMGAR10 & Ardila & LM & LM & HYB & HYB & + & KY930976 \\
\hline BMGAR2 & Ardila & LM & LS & HYB & HYB & + & KU368395 \\
\hline
\end{tabular}

Table continues on next page. 
Table 3 cont.

\begin{tabular}{|c|c|c|c|c|c|c|c|}
\hline \multirow{2}{*}{ Individual ID } & \multirow{2}{*}{ Sub-basin } & \multicolumn{5}{|c|}{ Identification criterion } & \multirow{2}{*}{$\begin{array}{c}\text { GenBank } \\
\text { Accession No }\end{array}$} \\
\hline & & Field ID & Cytb ID & Beta-actin ID & Mito-nuclear ID & Morphometry & \\
\hline BMGAR3 & Ardila & LM & LS & LS & LS & + & KU368396 \\
\hline BMGAR4 & Ardila & LM & LS & LS & LS & + & KU368397 \\
\hline BMGAR5 & Ardila & LM & LS & LS & LS & + & KU368398 \\
\hline BMGAR6 & Ardila & LM & LS & HYB & HYB & + & KU368399 \\
\hline BMGAR7 & Ardila & LM & LS & LS & LS & & KU368400 \\
\hline BMGAR8 & Ardila & LM & $\mathrm{LC}$ & LS & HYB & + & KU368243 \\
\hline BMGAR9 & Ardila & LM & $\mathrm{LC}$ & HYB & HYB & + & KU368244 \\
\hline BMGCH1 & Chança & LM & $\mathrm{LS}$ & LS & LS & + & KU368410 \\
\hline BMGCH10 & Chança & LM & LS & HYB & HYB & + & KU368411 \\
\hline BMGCH11 & Chança & LM & $\mathrm{LC}$ & LS & HYB & + & KU368257 \\
\hline BMGCH12 & Chança & LM & LS & $\mathrm{LC}$ & HYB & + & KU368412 \\
\hline $\mathrm{BMGCH} 2$ & Chança & LM & LS & HYB & HYB & + & KU368414 \\
\hline BMGCH3 & Chança & LM & $\mathrm{LC}$ & LS & HYB & + & KU368258 \\
\hline BMGCH4 & Chança & LM & $\mathrm{LS}$ & LS & LS & + & KY930977 \\
\hline BMGCH5 & Chança & LM & LS & HYB & HYB & + & KU368415 \\
\hline BMGCH6 & Chança & LM & LS & $\mathrm{LC}$ & HYB & + & KU368416 \\
\hline BMGCH7 & Chança & LM & $\mathrm{LC}$ & $\mathrm{LC}$ & $\mathrm{LC}$ & + & KU368259 \\
\hline BMGCH8 & Chança & LM & $\mathrm{LS}$ & LS & LS & + & KU368417 \\
\hline BMGCH9 & Chança & LM & LS & $\mathrm{LC}$ & HYB & + & KU368418 \\
\hline BMGO1 & Oeiras & LM & LM & LM & LM & & KY930978 \\
\hline BMGO2 & Oeiras & LM & LM & LM & LM & & KY930979 \\
\hline BMGO3 & Oeiras & LM & LM & HYB & HYB & & KY930980 \\
\hline BMGV1 & Vascão & LM & LS & LS & LS & + & KU368491 \\
\hline BMGV10 & Vascão & LM & $\mathrm{LS}$ & LS & LS & + & KY930981 \\
\hline BMGV11 & Vascão & LM & LS & LS & LS & + & KU368492 \\
\hline BMGV12 & Vascão & LM & LS & LS & LS & + & KU368493 \\
\hline BMGV13 & Vascão & LM & LS & LS & LS & & KU368494 \\
\hline BMGV2 & Vascão & LM & LS & $\mathrm{LC}$ & HYB & + & KU368495 \\
\hline BMGV3 & Vascão & LM & LS & HYB & HYB & + & KU368496 \\
\hline BMGV4 & Vascão & LM & $\mathrm{LS}$ & HYB & HYB & + & KU368497 \\
\hline BMGV5 & Vascão & LM & LS & $\mathrm{LC}$ & HYB & + & KU368498 \\
\hline BMGV6 & Vascão & LM & LS & LS & LS & + & KU368499 \\
\hline BMGV7 & Vascão & LM & LS & HYB & HYB & + & KU368500 \\
\hline BMGV8 & Vascão & LM & $\mathrm{LC}$ & HYB & HYB & + & KU368322 \\
\hline BMGV9 & Vascão & LM & LS & LS & LS & + & KU368502 \\
\hline BSG9 & Oeiras & LS & LM & HYB & HYB & & KY930982 \\
\hline BSGAR1 & Ardila & LS & LM & HYB & HYB & + & KY930983 \\
\hline BSGAR10 & Ardila & LS & LS & $\mathrm{LC}$ & HYB & + & KU368401 \\
\hline BSGAR11 & Ardila & LS & LM & LM & LM & & KY930984 \\
\hline BSGAR2 & Ardila & LS & $\mathrm{LC}$ & LS & HYB & + & KU368245 \\
\hline BSGAR3 & Ardila & LS & LM & HYB & HYB & + & KY930985 \\
\hline BSGAR4 & Ardila & LS & LS & LS & LS & + & KU368402 \\
\hline BSGAR6 & Ardila & LS & $\mathrm{LC}$ & LS & HYB & + & KU368247 \\
\hline BSGAR7 & Ardila & LS & $\mathrm{LC}$ & $\mathrm{LC}$ & $\mathrm{LC}$ & + & KU368248 \\
\hline BSGAR8 & Ardila & LS & $\mathrm{LC}$ & LS & HYB & + & KU368249 \\
\hline BSGAR9 & Ardila & LS & LM & LM & LM & + & KY930986 \\
\hline BSGCH1 & Chança & LS & $\mathrm{LC}$ & HYB & HYB & & KU368260 \\
\hline BSGCH13 & Chança & $\mathrm{LS}$ & $\mathrm{LC}$ & LS & HYB & & KU368261 \\
\hline BSGCH17 & Chança & LS & LS & LS & LS & + & KU368421 \\
\hline BSGCH18 & Chança & LS & $\mathrm{LC}$ & LS & HYB & + & KU368262 \\
\hline BSGCH19 & Chança & $\mathrm{LS}$ & LS & HYB & HYB & + & KU368422 \\
\hline BSGCH2 & Chança & LS & LS & $\mathrm{LC}$ & HYB & + & KU368423 \\
\hline BSGCH21 & Chança & LS & $\mathrm{LC}$ & LS & HYB & + & KU368264 \\
\hline BSGCH23 & Chança & $\mathrm{LS}$ & $\mathrm{LC}$ & LS & HYB & + & KY930987 \\
\hline
\end{tabular}


Table 3 cont.

\begin{tabular}{|c|c|c|c|c|c|c|c|}
\hline \multirow{2}{*}{ Individual ID } & \multirow{2}{*}{ Sub-basin } & \multicolumn{5}{|c|}{ Identification criterion } & \multirow{2}{*}{$\begin{array}{c}\text { GenBank } \\
\text { Accession No }\end{array}$} \\
\hline & & Field ID & Cytb ID & Beta-actin ID & Mito-nuclear ID & Morphometry & \\
\hline BSGCH24 & Chança & LS & $\mathrm{LC}$ & $\mathrm{LC}$ & $\mathrm{LC}$ & + & KU368267 \\
\hline BSGCH25 & Chança & LS & $\mathrm{LC}$ & LS & HYB & + & KU368268 \\
\hline BSGCH26 & Chança & LS & LS & LS & LS & + & KU368424 \\
\hline BSGCH27 & Chança & LS & LM & LS & HYB & + & KY930988 \\
\hline $\mathrm{BSGCH} 28$ & Chança & LS & $\mathrm{LC}$ & LS & HYB & + & KU368269 \\
\hline BSGCH29 & Chança & LS & LM & HYB & HYB & + & KY930989 \\
\hline BSGCH30 & Chança & LS & LM & HYB & HYB & + & KY930990 \\
\hline BSGCH31 & Chança & LS & LS & $\mathrm{LC}$ & HYB & + & KU368425 \\
\hline BSGCH32 & Chança & LS & LS & LS & LS & + & KU368426 \\
\hline BSGCH33 & Chança & LS & LS & LS & LS & + & KU368427 \\
\hline BSGCH34 & Chança & LS & $\mathrm{LC}$ & HYB & HYB & + & KU368270 \\
\hline BSGCH35 & Chança & LS & LS & LS & LS & + & KU368428 \\
\hline BSGCH36 & Chança & LS & LS & LS & LS & + & KU368429 \\
\hline BSGCH37 & Chança & LS & $\mathrm{LC}$ & HYB & HYB & + & KU368271 \\
\hline BSGCH38 & Chança & LS & LS & HYB & HYB & + & KU368430 \\
\hline BSGCH39 & Chança & LS & $\mathrm{LC}$ & HYB & HYB & + & KU368272 \\
\hline BSGCH40 & Chança & LS & LM & HYB & HYB & + & KY930991 \\
\hline BSGCH42 & Chança & LS & LS & LS & LS & + & KU368433 \\
\hline BSGCH43 & Chança & LS & $\mathrm{LC}$ & HYB & HYB & + & KU368273 \\
\hline BSGCH44 & Chança & LS & LM & LM & LM & + & KY930992 \\
\hline BSGCH46 & Chança & LS & LM & LM & LM & & KY930993 \\
\hline BSGCH9 & Chança & LS & LS & LS & LS & + & KU368436 \\
\hline BSGCO1 & Cobres & LS & LM & HYB & HYB & + & KY930994 \\
\hline BSGO1 & Oeiras & LS & LM & LM & LM & + & KY930995 \\
\hline BSGO3 & Oeiras & LS & LM & LM & LM & + & KY930996 \\
\hline BSGO4 & Oeiras & LS & $\mathrm{LC}$ & HYB & HYB & + & KY930997 \\
\hline BSGO5 & Oeiras & LS & $\mathrm{LC}$ & LS & HYB & + & KY930998 \\
\hline BSGO6 & Oeiras & LS & LM & LM & LM & + & KY930999 \\
\hline BSGO8 & Oeiras & LS & $\mathrm{LC}$ & HYB & HYB & & KY931000 \\
\hline BSGO9 & Oeiras & LS & LM & HYB & HYB & + & KY931001 \\
\hline BSGOD1 & Odeleite & LS & $\mathrm{LC}$ & HYB & HYB & + & KY931002 \\
\hline BSGOD14 & Odeleite & LS & LS & LS & LS & + & KU368448 \\
\hline BSGOD15 & Odeleite & LS & LS & LS & LS & + & KU368449 \\
\hline BSGOD16 & Odeleite & LS & LS & LS & LS & + & KY931003 \\
\hline BSGOD20 & Odeleite & LS & LM & HYB & HYB & + & KY931004 \\
\hline BSGOD6 & Odeleite & LS & LM & $\mathrm{LC} / \mathrm{LB}$ & HYB & + & KY931005 \\
\hline BSGV1 & Vascão & LS & $\mathrm{LC}$ & LS & HYB & + & KU368323 \\
\hline BSGV19 & Vascão & LS & LS & LS & LS & + & KU368514 \\
\hline BSGV3 & Vascão & LS & $\mathrm{LC}$ & $\mathrm{LC}$ & $\mathrm{LC}$ & & KU368329 \\
\hline BSGV4 & Vascão & LS & LS & $\mathrm{LC}$ & HYB & + & KU368524 \\
\hline BSGV5 & Vascão & LS & LS & LS & LS & + & KU368519 \\
\hline BSGV53 & Vascão & LS & LS & HYB & HYB & + & KY931006 \\
\hline BSGV54 & Vascão & LS & LS & LS & LS & + & KU368510 \\
\hline BSGV56 & Vascão & LS & LS & HYB & HYB & + & KU368508 \\
\hline BSGV57 & Vascão & LS & LS & LS & LS & + & KU368507 \\
\hline BSGV58 & Vascão & LS & LS & LS & LS & + & KU368523 \\
\hline BSGV59 & Vascão & LS & $\mathrm{LC}$ & HYB & HYB & + & KU368333 \\
\hline BSGV60 & Vascão & LS & LS & LS & LS & + & KU368506 \\
\hline BSGV61 & Vascão & LS & LS & HYB & HYB & + & KU368505 \\
\hline BSGV62 & Vascão & LS & LS & HYB & HYB & + & KU368522 \\
\hline BSGV64 & Vascão & LS & LS & $\mathrm{LC}$ & HYB & + & KU368504 \\
\hline BSGV65 & Vascão & LS & $\mathrm{LC}$ & HYB & HYB & + & KU368332 \\
\hline BSGV71 & Vascão & LS & $\mathrm{LC}$ & LS & HYB & & KU368330 \\
\hline BSGV73 & Vascão & LS & LS & LS & LS & & KU368518 \\
\hline
\end{tabular}


Table 3 cont.

\begin{tabular}{|c|c|c|c|c|c|c|c|}
\hline \multirow{2}{*}{ Individual ID } & \multirow{2}{*}{ Sub-basin } & \multicolumn{5}{|c|}{ Identification criterion } & \multirow{2}{*}{$\begin{array}{c}\text { GenBank } \\
\text { Accession No }\end{array}$} \\
\hline & & Field ID & Cytb ID & Beta-actin ID & Mito-nuclear ID & Morphometry & \\
\hline BSGV8 & Vascão & $\mathrm{LS}$ & $\mathrm{LS}$ & $\mathrm{LS}$ & $\mathrm{LS}$ & & KU368525 \\
\hline BSTGAR10 & Ardila & LST & $\mathrm{LC}$ & $\mathrm{LC}$ & $\mathrm{LC}$ & + & KU368250 \\
\hline BSTGAR11 & Ardila & LST & LS & LS & LS & + & KU368403 \\
\hline BSTGAR12 & Ardila & LST & $\mathrm{LC}$ & HYB & HYB & + & KU368251 \\
\hline BSTGAR13 & Ardila & LST & $\mathrm{LC}$ & HYB & HYB & + & KY931007 \\
\hline BSTGAR3 & Ardila & LST & $\mathrm{LC}$ & $\mathrm{LC}$ & $\mathrm{LC}$ & + & KY931008 \\
\hline BSTGAR4 & Ardila & LST & $\mathrm{LC}$ & LS & HYB & + & KU368254 \\
\hline BSTGAR6 & Ardila & LST & LS & HYB & HYB & + & KU368407 \\
\hline BSTGAR7 & Ardila & LST & LS & LS & LS & + & KU368408 \\
\hline BSTGAR8 & Ardila & LST & $\mathrm{LC}$ & LS & HYB & + & KU368255 \\
\hline BSTGAR9 & Ardila & LST & LS & HYB & HYB & & KU368409 \\
\hline BSTGO11 & Oeiras & LST & LM & LM & LM & + & KY931009 \\
\hline BSTGO13 & Oeiras & LST & LM & LM & LM & + & KY931010 \\
\hline BSTGO3 & Oeiras & LST & LM & LM & LM & + & KY931011 \\
\hline BSTGO4 & Oeiras & LST & LM & HYB & HYB & + & KY931012 \\
\hline BSTGO5 & Oeiras & LST & LM & LM & LM & + & KY931013 \\
\hline BSTGOD1 & Odeleite & LST & LS & $\mathrm{LC}$ & HYB & + & KU368457 \\
\hline BSTGOD10 & Odeleite & LST & $\mathrm{LC}$ & LS & HYB & + & KY931014 \\
\hline BSTGOD11 & Odeleite & LST & LS & LS & LS & + & KU368458 \\
\hline BSTGOD12 & Odeleite & LST & $\mathrm{LC}$ & LS & HYB & + & KY931015 \\
\hline BSTGOD13 & Odeleite & LST & LS & LS & LS & + & KU368459 \\
\hline BSTGOD14 & Odeleite & LST & $\mathrm{LC}$ & LS & HYB & + & KY931016 \\
\hline BSTGOD15 & Odeleite & LST & LS & LS & LS & + & KU368460 \\
\hline BSTGOD16 & Odeleite & LST & LS & LS & LS & + & KY931017 \\
\hline BSTGOD18 & Odeleite & LST & LS & LS & LS & + & KU368461 \\
\hline BSTGOD19 & Odeleite & LST & LS & HYB & HYB & + & KU368462 \\
\hline BSTGOD2 & Odeleite & LST & LS & LS & LS & + & KU368463 \\
\hline BSTGOD3 & Odeleite & LST & LS & LS & LS & + & KU368464 \\
\hline BSTGOD4 & Odeleite & LST & LS & LS & LS & + & KY931018 \\
\hline BSTGOD5 & Odeleite & LST & $\mathrm{LC}$ & LS & HYB & + & KY931019 \\
\hline BSTGOD6 & Odeleite & LST & LS & LS & LS & + & KU368465 \\
\hline BSTGOD7 & Odeleite & LST & $\mathrm{LC}$ & LS & HYB & + & KY931020 \\
\hline BSTGOD8 & Odeleite & LST & LS & $\mathrm{LC}$ & HYB & + & KU368466 \\
\hline BSTGOD9 & Odeleite & LST & LS & LS & LS & + & KU368467 \\
\hline
\end{tabular}

Identification criteria: Field ID = field identification, Cyt $b$ ID $=$ mitochondrial cytochrome $b$ gene sequencing, Beta-actin ID $=$ nuclear betaactin gene sequencing, Mito-nuclear ID = combined information retrieved from the sequencing of the cyt $b$ and beta-actin genes; Individuals used in morphometric analyses are marked with a plus sign $(+)$; GenBank accession numbers of cyt $b$ gene sequences are also included; Legend: $\mathrm{LC}=$ L. comizo, $\mathrm{LS}=$ L. sclateri, $\mathrm{LM}=$ L. microcephalus, $\mathrm{LST}=$ L. steindachneri, $\mathrm{HYB}=\mathrm{hybrid}, \mathrm{LC} / \mathrm{LB}=\mathrm{a}$ mixture of $L$. comizo and L. bocagei species-specific tags.

position of the fins (Almaça 1967, Doadrio et al. 2011). The use of geometric morphometrics, which is proven to be reliable for the distinction of Barbus species (Geiger et al. 2016), was not possible due to the impossibility of adequately collect landmarks in the field, since sampling was directed to collect fin clips for population genetic studies under the scope of the FISHATLAS project (Sousa-Santos et al. 2016). Thus, and although the main goal of the present paper was to assess the reliability of in loco species identification (commonly used in technical reports and environmental impact studies), we decided to use photographs made in the field to take traditional morphometric measures and further test if a simple and expedite methodological procedure as such, which could be conducted by less experienced technicians, would be reliable for species differentiation.
Statistical analyses. To explore the relation between the genetic identity of the Guadiana barbels and the morphological variables studied we used raw data residuals of log-log regressions of the morphometric variables, using the head size (distance between the anterior limit of the head and the posterior limit of the operculum) as an independent variable. The variables SLLD (a meristic variable) and ANG (an angular measure) were not transformed. To perform a principal component analysis (PCA) and to maximize the number of individuals included (due to the high number of missing values), twelve variables were selected (the residuals concerning LDE, TDE, PEPO, MHD, TSAD, TSAP, TSAV, DP, $\mathrm{DV}, \mathrm{PV}, \mathrm{DPO}$, and $\mathrm{ANG})$. This later variable (ANG) was transformed by a logarithmic function to improve normality. Five extreme outliers were extracted from the 
analysis, which was conducted with the remaining 152 a discriminant analysis was performed using the same individuals. Both graphic visual inspections and values $\log$-log residuals of the genetically pure $L$. sclateri and of kurtosis and skewness (between -2 and 2) suggest that L. comizo (for L. microcephalus the number of individuals these variables have approximately a normal distribution. with morphological measures was very restricted). Kaiser-Meyer-Olkin measure of sampling adequacy and The discriminant function was then used to classify the Bartlett's test of Sphericity were used to evaluate the genetically hybrid individuals. All statistical analyses suitability of the PCA analysis to this dataset. A PCA were conducted using IBM SPSS Statistics, version 22. analysis was performed using the correlation matrix. The presently reported study was carried out in The relation between the mito-nuclear genetic identity accordance with the Portuguese state regulations and of individuals and the morphological analysis was necessary permits to conduct fieldwork were required to examined using scatterplot graphic concerning the PCA the National Institute for the Conservation of Nature and components, here treated as new variables. Additionally, Forests, Portugal.

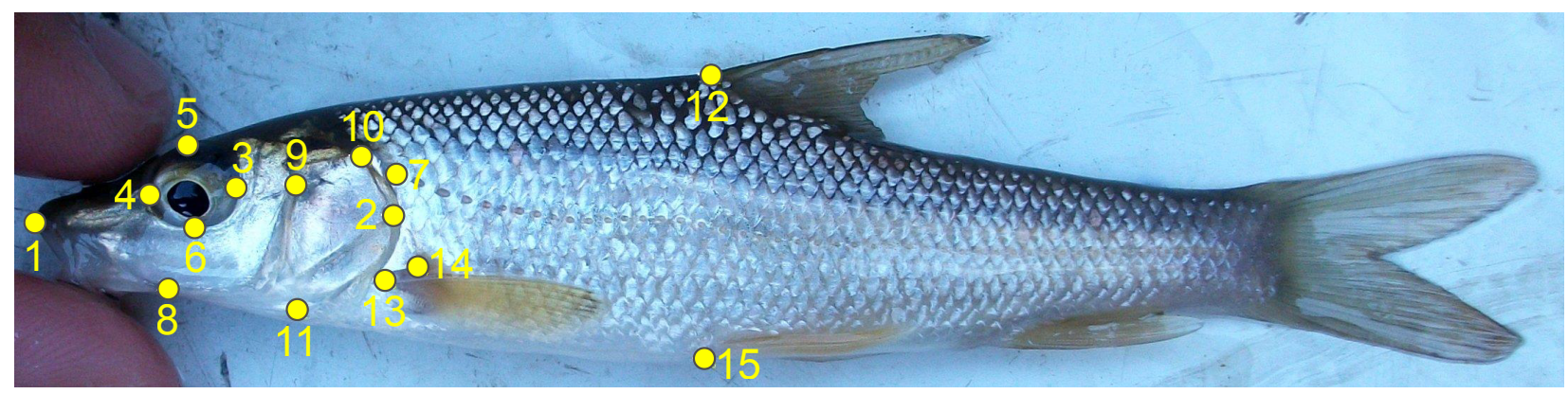

Fig. 3. Location of the 15 morphometric landmarks used for morphometric analyses; legend: $1=$ tip of the snout, $2=$ posterior edge of the operculum, $3=$ posterior edge of the eye, $4=$ anterior edge of the eye, $5=$ superior edge of the eye, $6=$ inferior edge of the eye, $7=$ first scale of the lateral line, $8=$ posterior end of the maxilla, $9=$ anterior edge of the operculum, $10=$ dorsal edge of the operculum, $11=$ ventral edge of the operculum, $12=$ anterior insertion of the dorsal fin, 13 = anterior insertion of the pectoral fin, $14=$ posterior insertion of the pectoral fin, $15=$ anterior insertion of the ventral fin

Table 4

Description of the 19 measures used as variables for morphometric analyses and the respective landmarks used to extract them, when applicable; the numbers in the first column corresponding to the landmarks depicted in Fig. 3

\begin{tabular}{ccl}
\hline \multirow{2}{*}{ Landmarks } & & \multicolumn{1}{c}{ Variable } \\
\cline { 2 - 3 } & Acronym & \multicolumn{1}{c}{ Description } \\
\hline $1-2$ & TSPO & Distance from tip of snout until posterior edge of operculum \\
$1-3$ & TSPE & Distance from tip of snout until posterior edge of eye \\
$3-4$ & LDE & Longitudinal diameter of eye \\
$5-6$ & TDE & Transversal diameter of eye \\
$3-2$ & PEPO & Distance from posterior edge of eye until posterior edge of operculum \\
$1-8$ & ML & Maxilla length \\
$1-7$ & TSLL & Distance from tip of snout until beginning of lateral line \\
$1-9$ & TPAO & Distance from tip of snout until anterior edge of operculum \\
$10-11$ & MHO & Maximum height between dorsal and ventral edges of operculum \\
$1-12$ & TSAD & Distance from tip of snout until anterior insertion of dorsal fin \\
$1-13$ & TSAP & Distance from tip of snout until anterior insertion of pectoral fin \\
$1-15$ & TSAV & Distance from tip of snout until anterior insertion of ventral fin \\
$12-13$ & DP & Distance between anterior insertions of dorsal and pectoral fins \\
$12-15$ & DV & Distance between anterior insertions of dorsal and ventral fins \\
$13-15$ & PV & Distance between anterior insertions of pectoral and ventral fins \\
- & SLLD & Number of scales, counted perpendicularly, from beginning of lateral line until \\
& anterior insertion of dorsal fin \\
$12-2$ & DPO & Distance from anterior insertion of dorsal fin until posterior edge of operculum \\
$10-11$ & ANG & Angle formed by intersection between line drawn between dorsal and ventral edges \\
and & & of operculum and line drawn between anterior and posterior edges of operculum \\
$10-9$ & DPH & Dorsal profile of head: concave (0) or convex (1) \\
- & &
\end{tabular}




\section{RESULTS}

Mitochondrial and nuclear genotyping. A total of 320 and 226 Luciobarbus adult specimens from the Guadiana River were sequenced for the cyt $b$ and beta-actin genes, respectively. Provisional species identifications based only on mtDNA results retrieved 141 L. sclateri, 135 L. comizo, and 44 L. microcephalus while that based on the beta-actin gene sequences retrieved 81 L. sclateri, 53 L. comizo, 20 L. microcephalus, and 72 hybrids (Table 5).

Among nDNA sequences, 42 diagnostic single nucleotide polymorphisms (SNPs) were identified (Table 6). For the majority of the cases, instead of single peaks, two or three bases were found superimposed in each locus (represented in Table 6 by the respective ambiguity codes).

The analysis of the SNPs patterns (Table 6), corroborated with the mtDNA results and the sequencing of specimens from other river basins (for L. comizo and L. sclateri), revealed that at least six loci of the studied beta-actin gene fragment may be used as species-specific tags (Table 7). All the L. sclateri specimens from Guadiana and from the rivers Arade and Seixe (where the species occurs allopatrically) show the exclusive R-C-G-Y-R-S/B combination of nucleotides (Tables 6 and 7). Luciobarbus comizo from Guadiana differ from $L$ sclateri by a single diagnostic locus: a double peak of A and $\mathrm{G}$ nucleotides ( $\mathrm{R}$ code of ambiguity) is found in locus 117, instead of the single G peak found in L. sclateri specimens (Table 7). The L. comizo specific tag $(117 \mathrm{R})$ was validated by using specimens from the Tagus River $(n=51)$ : although no pure $L$. comizo individuals were detected, it was possible to detect the L. comizo specific tag in 11 L. bocage $\times$ L. comizo hybrids (these two species occur in sympatry in the Tagus). Moreover, these hybrids showed a triple peak (B ambiguity code) at locus 279 , corresponding to the mixture of the typical GC (S) of L. comizo with the typical GT (K) of L. bocagei (Table $6)$, reinforcing their identification as interspecific hybrids.

\section{Table 5}

Identification of Luciobarbus sampled in the Guadiana River sub-basins based on mtDNA only and on nDNA only

\begin{tabular}{|c|c|c|c|c|c|c|c|}
\hline \multirow[b]{2}{*}{ River basin } & \multicolumn{3}{|c|}{$\begin{array}{l}\text { mtDNA-based } \\
\text { identification }\end{array}$} & \multicolumn{4}{|c|}{$\begin{array}{l}\text { nDNA-based } \\
\text { identification }\end{array}$} \\
\hline & 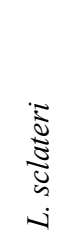 & 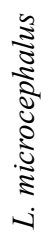 & 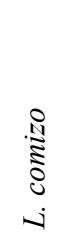 & 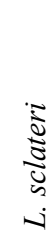 & 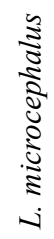 & $\begin{array}{c}0 \\
\stackrel{N}{\Xi} \\
0 \\
ن\end{array}$ & 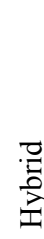 \\
\hline Caia & & 6 & 5 & & 3 & 1 & 6 \\
\hline Degebe & 4 & & 15 & & & 7 & 6 \\
\hline Ardila & 26 & 9 & 36 & 17 & 2 & 15 & 12 \\
\hline Chança & 33 & 6 & 24 & 20 & 2 & 7 & 13 \\
\hline Cobres & 4 & 1 & 22 & 1 & & 10 & 6 \\
\hline Oeiras & 9 & 20 & 7 & 4 & 13 & 2 & 12 \\
\hline Vascão & 34 & & 18 & 20 & & 7 & 14 \\
\hline Odeleite & 31 & 2 & 8 & 19 & & 4 & 3 \\
\hline Total & 141 & 44 & 135 & 81 & 20 & 53 & 72 \\
\hline
\end{tabular}

The SNP pattern of L. bocagei (R-C-R-Y-A-K) identified in specimens from the Tagus was validated by using 80 specimens of $L$. bocagei captured in six river basins where this species occurs allopatrically (Table 6). The beta-actin gene sequences of $L$. bocagei are extremely conserved: all these individuals, from the whole distribution area of the species, showed the same SNP pattern for the 42 analysed loci (Table 6). Interestingly, the typical L. bocagei SNP pattern was detected in one individual from the Guadiana bearing a L. microcephalus mtDNA (Table 6).

Regarding the target specimens from Guadiana, the majority of the individuals $(n=134,59.3 \%)$ belonged to a given Luciobarbus species (61 L. sclateri, 49 L. comizo, and $24 \mathrm{~L}$. microcephalus), confirmed by the analysis of their mtDNA and nDNA profiles, while the remaining individuals $(n=92 ; 40.7 \%)$ were interspecific hybrids. Among the hybrids, $51.1 \%(n=47)$ had a mixture of species-specific tags of more than one Luciobarbus species in their nuclear sequences, and $48.9 \%(n=45)$ exhibited mito-nuclear incongruence: the nDNA profile was typical of a Luciobarbus species which was distinct from that identified at the mtDNA level (Table 6). This latter group of interspecific hybrids (representing $33.6 \%$ of the total number of individuals sequenced) included $28 \mathrm{~L}$. sclateri with $L$. comizo mtDNA, 15 L. comizo with L. sclateri mtDNA, one L. sclateri with L. microcephalus mtDNA and one individual with $L$. comizo and $L$. bocagei nuclear species-specific tags bearing mtDNA of L. microcephalus (Table 6).

When considering the percentage of hybrids in each sub-basin, we found that in the majority of the subbasins the percentage of hybrids was below 35\% $(25.0 \%$ in Degebe, $27.3 \%$ in Cobres, $29.6 \%$ in Oeiras, $34.2 \%$ in Vascão, and $34.6 \%$ in Odeleite), while in the other subbasins these individuals were prevalent $(50.0 \%$ in Ardila, $54.8 \%$ in Chança, and $60.0 \%$ in Caia).

Taxonomical identification based on phenotypic and genetic data. Individual results of the phenotypic-, mtDNA-, and mito-nuclear-based assignments are presented in Table 6. From the 194 individuals identified in loco based on phenotypic traits (see Materials and Methods), $74(38.1 \%)$ were identified as L. sclateri (Table 8). The remaining three species, L. steindachneri, L. comizo, and L. microcephalus, accounted for $17.0 \%$ $23.2 \%$ of the total number of individuals (Table 8). No hybrids were phenotypically assigned (Table 8).

If only mtDNA data is considered for taxonomical identification, the most common species would still be L. sclateri, although its relative frequency was higher than the one obtained when phenotypic characters were used for in loco identification (44.3\% vs. 38.1\%) (Table 8). The relative frequency of $L$. comizo will also be higher $(38.7 \%$ vs. $21.7 \%$, respectively, for mtDNA-based and in loco identifications) but, contrastingly, the relative frequencies of L. microcephalus and L. steindachneri will be lower when using the identification based on mtDNA (17.0\% vs. $23.2 \%$ and $0 \%$ vs. $17.0 \%$, respectively) (Table 8 ).

If only nDNA is considered, a fourth taxonomical category emerged ("hybrids", with a relative frequency of 
Table 6

SNPs found in the analysed beta-actin gene fragment in the studied Luciobarbus fishes from Iberian rivers

\begin{tabular}{|c|c|c|c|c|c|c|c|c|c|c|c|c|c|c|c|c|c|}
\hline \multirow{2}{*}{\multicolumn{2}{|c|}{ Parameter }} & \multicolumn{16}{|c|}{ River basin } \\
\hline & & \multicolumn{9}{|c|}{ Guadiana } & \multirow{2}{*}{$\begin{array}{c}\begin{array}{c}\text { Arade } \\
\text { and } \\
\text { Seixe }\end{array} \\
13\end{array}$} & \multicolumn{3}{|l|}{ LBR } & \multicolumn{3}{|c|}{ Tagus } \\
\hline \multirow{4}{*}{\multicolumn{2}{|c|}{$\begin{array}{c}n \\
\text { mtDNA ID } \\
\text { nDNA ID } \\
\text { mt-n GNT }\end{array}$}} & 49 & 24 & 61 & 28 & 1 & 1 & 15 & 20 & 14 & & 26 & 80 & 24 & 16 & 7 & 4 \\
\hline & & $\mathrm{LC}$ & LM & LS & $\mathrm{LC}$ & LM & LM & LS & $\mathrm{LC}$ & LM & LS & LS & LB & LB & $\mathrm{LC}$ & LB & $\mathrm{LC}$ \\
\hline & & $\mathrm{LC}$ & LM & LS & LS & $\mathrm{CB}$ & LS & LC & Hybrid & Hybrid & Hybrid & LS & LB & LB & LB & LBxLC & LBxLC \\
\hline & & $\mathrm{LC}$ & LM & LS & \multicolumn{4}{|c|}{ HB-M } & \multicolumn{3}{|c|}{ HYB } & LS & LB & LB & HB-M & \multicolumn{2}{|c|}{ HYB } \\
\hline \multirow{6}{*}{ 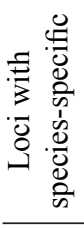 } & & $\mathrm{R}$ & A & $\mathrm{R}$ & $\mathrm{R}$ & $\mathrm{R}$ & $\mathrm{R}$ & $\mathrm{R}$ & $\mathrm{R} / \mathrm{A}$ & $\mathrm{R}$ & $\mathrm{R}$ & $\mathrm{R}$ & $\mathrm{R}$ & $\mathrm{R}$ & $\mathrm{R}$ & $\mathrm{R}$ & $\mathrm{R}$ \\
\hline & 60 & $\mathrm{C}$ & Y & $\mathrm{C}$ & $\mathrm{C}$ & $\mathrm{C}$ & $\mathrm{C}$ & $\mathrm{C}$ & $\mathrm{C} / \mathrm{Y}$ & $\mathrm{C} / \mathrm{Y}$ & $\mathrm{C} / \mathrm{Y}$ & $\mathrm{C}$ & $\mathrm{C}$ & $\mathrm{C}$ & $\mathrm{C}$ & $\mathrm{C}$ & $\mathrm{C}$ \\
\hline & 117 & $\mathrm{R}$ & $\mathrm{R}$ & G & G & $\mathrm{R}$ & G & $\mathrm{R}$ & $\mathrm{R} / \mathrm{G}$ & $\mathrm{R} / \mathrm{V} / \mathrm{G}$ & $\mathrm{R}$ & G & $\mathrm{R}$ & $\mathrm{R}$ & $\mathrm{R}$ & $\mathrm{R}$ & $\mathrm{R}$ \\
\hline & 126 & Y & $\mathrm{T}$ & Y & $\mathrm{Y}$ & $\mathrm{Y}$ & $\mathrm{Y}$ & $\mathrm{Y}$ & $\mathrm{Y} / \mathrm{T}$ & $\mathrm{Y} / \mathrm{H}$ & Y & $\mathrm{Y}$ & Y & Y & Y & Y & $\mathrm{Y}$ \\
\hline & 245 & $\mathrm{R}$ & G & $\mathrm{R}$ & $\mathrm{R}$ & A & $\mathrm{R}$ & $\mathrm{R}$ & $\mathrm{R}$ & $\mathrm{R}$ & $\mathrm{R}$ & $\mathrm{R}$ & A & A & A & $\mathrm{R}$ & $\mathrm{R}$ \\
\hline & 279 & $\mathrm{~S}$ & $\mathrm{~K} / \mathrm{D}$ & $\mathrm{S} / \mathrm{B}$ & $\mathrm{S}$ & $\mathrm{K}$ & $\mathrm{S}$ & $\mathrm{S}$ & $\mathrm{S} / \mathrm{B} / \mathrm{K}$ & $\mathrm{S} / \mathrm{B}$ & $\mathrm{S} / \mathrm{B}$ & $\mathrm{S}(\mathrm{GC})$ & $\mathrm{K}(\mathrm{GT})$ & $\mathrm{K}(\mathrm{GT})$ & $\mathrm{K}(\mathrm{GT})$ & $\mathrm{B}(\mathrm{GCT})$ & $\mathrm{B}(\mathrm{GCT})$ \\
\hline \multirow{36}{*}{ 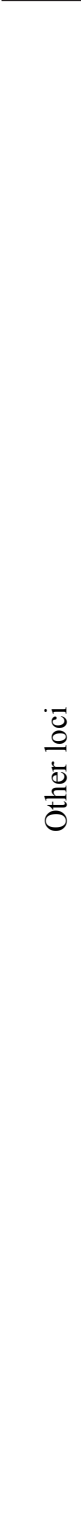 } & 181 & $\mathrm{R} / \mathrm{D}$ & $\mathrm{R}$ & $\mathrm{R}$ & $\mathrm{R}$ & $\mathrm{W}$ & $\mathrm{R}$ & $\mathrm{R}$ & $\mathrm{R} / \mathrm{D}$ & $\mathrm{R}$ & $\mathrm{R} / \mathrm{D}$ & $\mathrm{R}$ & $\mathrm{W}$ & W & W & $\mathrm{D}$ & D \\
\hline & 182 & $\mathrm{R} / \mathrm{D}$ & $\mathrm{R} / \mathrm{D}$ & $\mathrm{R}$ & $\mathrm{R}$ & $\mathrm{G}$ & $\mathrm{R}$ & $\mathrm{R}$ & $\mathrm{R} / \mathrm{D}$ & $\mathrm{R}$ & $\mathrm{R} / \mathrm{D} / \mathrm{A}$ & $\mathrm{R}$ & G & G & G & $\mathrm{R}$ & $\mathrm{R}$ \\
\hline & 183 & K & $\mathrm{K}$ & K & $\mathrm{K}$ & $\mathrm{R}$ & K & $\mathrm{K}$ & K & $\mathrm{K} / \mathrm{R}$ & $\mathrm{K} / \mathrm{D}$ & K & $\mathrm{R}$ & $\mathrm{R}$ & $\mathrm{R}$ & $\mathrm{D}$ & $\mathrm{D}$ \\
\hline & 184 & $\mathrm{~K} / \mathrm{B}$ & $\mathrm{K} / \mathrm{X}$ & $\mathrm{K} / \mathrm{X}$ & $\mathrm{K}$ & $\mathrm{K}$ & K & $\mathrm{K}$ & $\begin{array}{l}\mathrm{K} / \mathrm{B} / \\
\mathrm{D} / \mathrm{X}\end{array}$ & $\mathrm{K} / \mathrm{D}$ & K & K & K & $\mathrm{K}$ & $\mathrm{K}$ & $\mathrm{K}$ & $\mathrm{K}$ \\
\hline & 185 & $\mathrm{~T} / \mathrm{K}$ & $\mathrm{T}$ & $\mathrm{T}$ & $\mathrm{T}$ & $\mathrm{T}$ & $\mathrm{T}$ & $\mathrm{T}$ & $\mathrm{T} / \mathrm{K}$ & $\mathrm{T}$ & $\mathrm{T} / \mathrm{K}$ & $\mathrm{T}$ & $\mathrm{T}$ & $\mathrm{T}$ & $\mathrm{T}$ & $\mathrm{T}$ & $\mathrm{T}$ \\
\hline & 186 & $\mathrm{~K}$ & $\mathrm{~K} / \mathrm{B}$ & $\mathrm{K} / \mathrm{B}$ & K & K & K & $\mathrm{K}$ & $\mathrm{K} / \mathrm{B}$ & $\mathrm{K} / \mathrm{B}$ & K & K & K & $\mathrm{K}$ & K & K & $\mathrm{K}$ \\
\hline & 187 & $\mathrm{Y} / \mathrm{B}$ & $\mathrm{Y}$ & $\mathrm{Y}$ & $\mathrm{Y}$ & $\mathrm{T}$ & Y & $\mathrm{Y}$ & $\mathrm{Y} / \mathrm{B}$ & $\mathrm{Y} / \mathrm{C}$ & $\mathrm{Y} / \mathrm{S}$ & $\mathrm{Y}$ & $\mathrm{T}$ & $\mathrm{T}$ & $\mathrm{T}$ & Y & Y \\
\hline & 188 & $\mathrm{M} / \mathrm{V} / \mathrm{H}$ & $\mathrm{M} / \mathrm{H}$ & $\mathrm{M} / \mathrm{H}$ & $\mathrm{M}$ & $\mathrm{C}$ & M & $\mathrm{M}$ & $\mathrm{M} / \mathrm{H} / \mathrm{V}$ & $\mathrm{M} / \mathrm{H}$ & $\mathrm{M} / \mathrm{X} / \mathrm{H}$ & M & $\mathrm{C}$ & $\mathrm{C}$ & $\mathrm{C}$ & M & M \\
\hline & 189 & $\mathrm{R} / \mathrm{V}$ & $\mathrm{R} / \mathrm{V}$ & $\mathrm{R} / \mathrm{V}$ & $\mathrm{R}$ & A & $\mathrm{R}$ & $\mathrm{R}$ & $\begin{array}{l}\mathrm{R} / \mathrm{V} / \\
\mathrm{X} / \mathrm{D}\end{array}$ & $\mathrm{R} / \mathrm{X}$ & $\mathrm{R} / \mathrm{V}$ & $\mathrm{R}$ & A & $\mathrm{A} / \mathrm{R}$ & $\mathrm{A} / \mathrm{R}$ & $\mathrm{R}$ & $\mathrm{R}$ \\
\hline & 190 & $\mathrm{~K} / \mathrm{D}$ & $\mathrm{K} / \mathrm{D}$ & $\mathrm{K} / \mathrm{H}$ & $\mathrm{K}$ & $\mathrm{K}$ & K & K & $\mathrm{K} / \mathrm{D}$ & $\mathrm{K} / \mathrm{D}$ & $\mathrm{K} / \mathrm{D}$ & K & K & K & K & K & $\mathrm{K}$ \\
\hline & 191 & $\mathrm{G} / \mathrm{R} / \mathrm{K}$ & $\mathrm{G}$ & $\mathrm{G} / \mathrm{S} / \mathrm{K}$ & G & $\mathrm{G}$ & G & G & $\mathrm{G} / \mathrm{K}$ & G & $\mathrm{G} / \mathrm{K}$ & G & G & $\mathrm{G}$ & $\mathrm{G}$ & $\mathrm{G}$ & $\mathrm{G}$ \\
\hline & 192 & $\mathrm{~K} / \mathrm{B}$ & K & $\mathrm{K} / \mathrm{X}$ & $\mathrm{K}$ & $\mathrm{G}$ & K & K & $\mathrm{K} / \mathrm{B} / \mathrm{G}$ & K & $\mathrm{K} / \mathrm{D}$ & K & G & G & G & $\mathrm{K}$ & K \\
\hline & 193 & $\mathrm{~K} / \mathrm{D}$ & $\mathrm{K} / \mathrm{D}$ & $\mathrm{K} / \mathrm{B} / \mathrm{D}$ & $\mathrm{K}$ & $\mathrm{T}$ & K & $\mathrm{K}$ & $\begin{array}{c}\mathrm{K} / \mathrm{D} / \\
\mathrm{G} / \mathrm{R}\end{array}$ & $\mathrm{K} / \mathrm{D}$ & $\mathrm{K} / \mathrm{D} / \mathrm{G}$ & K & K & $\mathrm{T}$ & $\mathrm{T}$ & $\mathrm{K}$ & $\mathrm{K}$ \\
\hline & 194 & $\mathrm{~T}$ & $\mathrm{~T}$ & $\mathrm{~T}$ & $\mathrm{~T}$ & $\mathrm{~K}$ & $\mathrm{~T}$ & $\mathrm{~T}$ & $\mathrm{~T} / \mathrm{B} / \mathrm{K}$ & $\mathrm{T}$ & $\mathrm{T}$ & $\mathrm{T}$ & K & K & K & K & $\mathrm{K}$ \\
\hline & 195 & $\mathrm{~S} / \mathrm{B}$ & $\mathrm{S} / \mathrm{B}$ & S & $\mathrm{S}$ & $\mathrm{K}$ & S & $\mathrm{S}$ & $\begin{array}{l}\mathrm{S} / \mathrm{V} / \\
\mathrm{B} / \mathrm{D}\end{array}$ & $\mathrm{S}$ & $\mathrm{S} / \mathrm{B}$ & $\mathrm{S}$ & K & $\mathrm{K}$ & $\mathrm{K}$ & $\mathrm{B} / \mathrm{S}$ & $\mathrm{B} / \mathrm{S}$ \\
\hline & 196 & $\mathrm{R}$ & $\mathrm{R}$ & $\mathrm{R}$ & $\mathrm{R}$ & $\mathrm{S}$ & $\mathrm{R}$ & $\mathrm{R}$ & $\mathrm{R} / \mathrm{B} / \mathrm{D}$ & $\mathrm{R}$ & $\mathrm{R} / \mathrm{V}$ & $\mathrm{R}$ & $\mathrm{S}$ & $\mathrm{S}$ & $\mathrm{S}$ & V & V \\
\hline & 205 & $\mathrm{~A} / \mathrm{M}$ & A & A & A & $\mathrm{W}$ & A & A & $\mathrm{A} / \mathrm{R}$ & A & A & A & W & W & W & W & W \\
\hline & 206 & $\mathrm{~K} / \mathrm{D} / \mathrm{X}$ & K & $\mathrm{K} / \mathrm{B}$ & K & $\mathrm{R}$ & K & $\mathrm{K}$ & $\begin{array}{l}\mathrm{K} / \mathrm{D} / \\
\mathrm{B} / \mathrm{X}\end{array}$ & $\mathrm{K} / \mathrm{B}$ & $\mathrm{D} / \mathrm{K} / \mathrm{B}$ & K & $\mathrm{R}$ & $\mathrm{R}$ & $\mathrm{R}$ & D & D \\
\hline & 210 & $\mathrm{R} / \mathrm{D}$ & $\mathrm{R} / \mathrm{D}$ & $\mathrm{R} / \mathrm{D}$ & $\mathrm{R}$ & $\mathrm{G}$ & $\mathrm{R}$ & $\mathrm{R}$ & $\mathrm{D} / \mathrm{R}$ & $\mathrm{R} / \mathrm{D}$ & $\mathrm{R} / \mathrm{D}$ & $\mathrm{R}$ & G & G & G & $\mathrm{R}$ & $\mathrm{R}$ \\
\hline & 215 & $\mathrm{M} / \mathrm{V}$ & M & M & M & M & M & $\mathrm{M}$ & M/H/V & M & $\mathrm{M} / \mathrm{V}$ & M & M & M & M & M & M \\
\hline & 216 & $\mathrm{~K} / \mathrm{B}$ & $\mathrm{K}$ & $\mathrm{K} / \mathrm{B}$ & $\mathrm{K}$ & W & K & $\mathrm{K}$ & $\mathrm{K} / \mathrm{B}$ & $\mathrm{K} / \mathrm{B}$ & $\mathrm{K} / \mathrm{D} / \mathrm{B}$ & K & W & W & W & $\mathrm{D}$ & D \\
\hline & 232 & $\mathrm{~S} / \mathrm{V}$ & S & S & $\mathrm{S}$ & $\mathrm{Y}$ & S & $\mathrm{S}$ & $\mathrm{S}$ & $\mathrm{S}$ & S & S & Y & $\mathrm{Y}$ & $\mathrm{Y}$ & B & B \\
\hline & 233 & $\mathrm{~W} / \mathrm{H}$ & W & $\mathrm{W} / \mathrm{H}$ & W & $\mathrm{K}$ & W & $\mathrm{W}$ & $\mathrm{W} / \mathrm{H}$ & W & $\mathrm{W} / \mathrm{H}$ & W & $\mathrm{K}$ & $\mathrm{K}$ & $\mathrm{K}$ & $\mathrm{D}$ & $\mathrm{D}$ \\
\hline & 234 & $\mathrm{Y} / \mathrm{H} / \mathrm{B}$ & Y & $\mathrm{Y}$ & $\mathrm{Y}$ & $\mathrm{M}$ & $\mathrm{Y}$ & $\mathrm{Y}$ & $\mathrm{Y} / \mathrm{B}$ & Y & $\mathrm{Y}$ & $\mathrm{Y}$ & M & $\mathrm{M}$ & M & $\mathrm{H}$ & $\mathrm{H}$ \\
\hline & 235 & $\mathrm{R} / \mathrm{V}$ & $\mathrm{R}$ & $\mathrm{R} / \mathrm{D} / \mathrm{K}$ & $\mathrm{R}$ & W & $\mathrm{R}$ & $\mathrm{R}$ & $\mathrm{R} / \mathrm{D} / \mathrm{V}$ & $\mathrm{R}$ & $\mathrm{R} / \mathrm{D} / \mathrm{V}$ & $\mathrm{R}$ & W & W & W & $\mathrm{D}$ & $\mathrm{D}$ \\
\hline & 236 & $\mathrm{R} / \mathrm{V}$ & $\mathrm{R}$ & $\mathrm{R} / \mathrm{K}$ & $\mathrm{R}$ & $\mathrm{G}$ & $\mathrm{R}$ & $\mathrm{R}$ & $\mathrm{R} / \mathrm{A} / \mathrm{V}$ & $\mathrm{R}$ & $\mathrm{R} / \mathrm{A}$ & $\mathrm{R}$ & G & $\mathrm{G}$ & $\mathrm{G}$ & $\mathrm{R}$ & $\mathrm{R}$ \\
\hline & 237 & $\mathrm{R}$ & $\mathrm{R}$ & $\mathrm{R} / \mathrm{K}$ & $\mathrm{R}$ & A & $\mathrm{R}$ & $\mathrm{R}$ & $\mathrm{R} / \mathrm{D} / \mathrm{V}$ & $\mathrm{R}$ & $\mathrm{R} / \mathrm{X}$ & $\mathrm{R}$ & A & A & A & $\mathrm{R}$ & $\mathrm{R}$ \\
\hline & 238 & $\mathrm{G} / \mathrm{S} / \mathrm{R}$ & G & G & $\mathrm{G}$ & $\mathrm{G}$ & G & $\mathrm{G}$ & $\mathrm{G} / \mathrm{R} / \mathrm{S}$ & G & G & G & G & G & $\mathrm{G}$ & $\mathrm{G}$ & $\mathrm{G}$ \\
\hline & 239 & $\mathrm{C} / \mathrm{S} / \mathrm{V}$ & $\mathrm{C}$ & $\mathrm{C} / \mathrm{M}$ & $\mathrm{C}$ & $\mathrm{S}$ & $\mathrm{C}$ & $\mathrm{C}$ & $\mathrm{C} / \mathrm{M} / \mathrm{S}$ & $\mathrm{C}$ & $\mathrm{C} / \mathrm{S}$ & $\mathrm{C}$ & $\mathrm{S}$ & $\mathrm{S}$ & S & $\mathrm{S}$ & $\mathrm{S}$ \\
\hline & 240 & $\mathrm{M} / \mathrm{V}$ & $\mathrm{M}$ & $\mathrm{M} / \mathrm{H}$ & $\mathrm{M}$ & $\mathrm{M}$ & $\mathrm{M}$ & $\mathrm{M}$ & $\mathrm{M} / \mathrm{H} / \mathrm{V}$ & M & $\mathrm{M}$ & $\mathrm{M}$ & M & M & M & M & $\mathrm{M}$ \\
\hline & 241 & $\mathrm{M} / \mathrm{H}$ & $\mathrm{M}$ & $\mathrm{M} / \mathrm{H}$ & $\mathrm{M}$ & $\mathrm{M}$ & $\mathrm{M}$ & $\mathrm{M}$ & $\mathrm{M} / \mathrm{H}$ & M & M & M & M & M & M & M & M \\
\hline & 242 & $\mathrm{R}$ & $\mathrm{R}$ & $\mathrm{R} / \mathrm{V}$ & $\mathrm{R}$ & $\mathrm{S}$ & $\mathrm{R}$ & $\mathrm{R}$ & $\mathrm{R}$ & $\mathrm{R}$ & $\mathrm{R}$ & $\mathrm{R}$ & $\mathrm{S}$ & $\mathrm{S}$ & S & V & V \\
\hline & 243 & $\mathrm{R} / \mathrm{M}$ & $\mathrm{R}$ & $\mathrm{R} / \mathrm{D}$ & $\mathrm{R}$ & A & $\mathrm{R}$ & $\mathrm{R}$ & $\mathrm{R} / \mathrm{M}$ & $\mathrm{R}$ & $\mathrm{R}$ & $\mathrm{R}$ & A & A & A & $\mathrm{R}$ & $\mathrm{R}$ \\
\hline & 244 & $\mathrm{R}$ & $\mathrm{R}$ & $\mathrm{R} / \mathrm{V}$ & $\mathrm{R}$ & G & $\mathrm{R}$ & $\mathrm{R}$ & $\mathrm{R}$ & $\mathrm{R}$ & $\mathrm{R} / \mathrm{V}$ & $\mathrm{R}$ & G & G & $\mathrm{G}$ & $\mathrm{R}$ & $\mathrm{R}$ \\
\hline & 246 & $\mathrm{~S} / \mathrm{V}$ & S & S & $\mathrm{S}$ & $\mathrm{G}$ & S & $\mathrm{S}$ & $\mathrm{S} / \mathrm{V}$ & S & $\mathrm{S} / \mathrm{V}$ & S & G & $\mathrm{G}$ & $\mathrm{G}$ & S & $\mathrm{S}$ \\
\hline & 247 & $\mathrm{R}$ & $\mathrm{R}$ & $\mathrm{R}$ & $\mathrm{R}$ & $\mathrm{S}$ & $\mathrm{R}$ & $\mathrm{R}$ & $\mathrm{R}$ & $\mathrm{R}$ & $\mathrm{R}$ & $\mathrm{R}$ & $\mathrm{S}$ & $\mathrm{S}$ & $\mathrm{S}$ & $\mathrm{V}$ & V \\
\hline
\end{tabular}

Nucleotides or ambiguity codes (in the case of intra- and interspecific hybrids) are indicated for each loci. The first six loci listed were considered to be species-specific tags and are highlighted in different colours (blue for L. microcephalus, green for L. sclateri, orange for $L$. comizo from Guadiana, and purple for $L$. bocagei from Tagus). The provisional identification of individuals based on mtDNA is also indicated. $\mathrm{mt}-\mathrm{n}$ GNT $=$ mito-nuclear genotype, $\mathrm{LC}=$ L. comizo, $\mathrm{LM}=$ L. microcephalus, $\mathrm{LS}=L$. sclateri, $\mathrm{LB}=L$. bocagei, $\mathrm{HYB}=$ interspecific hybrids (individuals with a mixture of SNPs which are specific of two or more Luciobarbus species), HB-M = interspecific hybrids with mito-nuclear incongruence (individuals with mtDNA and nDNA of different Luciobarbus species), $\mathrm{LBR}=$ includes specimens from populations where only $L$. bocagei occurs (Ave, Vouga, Mondego, Lis, Alcoa, and Colares; see Fig. 2 for locations), CB = Individual with a mixture of L. comizo and L. bocagei nuclear species-specific tags although it was captured in the Guadiana, where L. bocagei is absent. 
$29.9 \%$ ) but the ordination of the relative frequencies of the three species is identical to that described for mtDNAbased assignments, although with lower percentages of each species (Table 8).

Finally, when mtDNA and nDNA data are combined to assign a mito-nuclear genotype to the individuals identified in loco, the results showed that the relative frequencies of L. comizo, L. sclateri, and L. microcephalus were overestimated when the identification was merely based on phenotypic traits and that $40.7 \%(n=79)$ of the individuals are in fact hybrids (Table 9), mothered mostly by $L$. comizo females $(50.6 \%, n=40)$ but also by females of L. sclateri $(34.2 \%, n=27)$ and of L. microcephalus $(15.2 \%, n=12)$ (Table 6).

Globally, regarding misidentifications, 62.4\% $(n=$ 121) of the in loco identifications were not concordant with the classifications based only on mtDNA. When the classifications are based on the mito-nuclear genotypes, the percentage of misidentifications increases to $82.0 \%$ ( $n=$ 159) (Table 9). More specifically, specimens phenotypically assigned to L. steindachneri were mostly pure individuals of one of the other three Luciobarbus species (13 L. sclateri, 4 L. microcephalus, and 2 L. comizo), while the remaining specimens were hybrids $(n=14)$ (Table 9). Contrastingly, concerning the other Luciobarbus species, the individuals wrongly identified in loco were mostly hybrids (Table 9). L. steindachneri and L. microcephalus were the species with the highest percentages of wrong classifications and L. comizo with the lowest (Table 9).

Concordant results between in loco identifications and mito-nuclear genotypes varied with the sub-basin considered: 15.4\% in Odeleite, $18.9 \%$ in Ardila, $21.9 \%$ in Chança, $26.1 \%$ in Oeiras, 39.0\% in Vascão, and 63.6\% in Cobres.

PCA on morphological analyses. The Principal Component Analysis (PCA) performed using the twelve variables for 152 individuals retained four components, all presenting eigenvalues higher than 1 . These four new variables created explained $83.3 \%$ of the variance. The visual inspection of the scatterplots relating these variables shows no clear separation between genetic entities (Fig. 4).

A discriminant analysis using two groups-L. sclateri and L. comizo - using the same variables selected above yielded highly significant results (Wilks' Lambda $=0.600$, $\left.X_{(12)}^{2}=40.931, P<0.001\right) .83 \%$ of the individuals were correctly assigned to their species ( $85 \%$ of the $L$. sclateri and $80 \%$ of the $L$. comizo were correctly classified). The same discriminant function applied to the hybrid individuals ( $n=$ 67) showed that they were mainly classified as L. sclateri $(71.6 \%)$, while the remaining $28.4 \%$ were classified as L. comizo.

\section{DISCUSSION}

Species identification in the field, in particular concerning freshwater fish species with subtle morphological diagnostic features, may impose considerable difficulties even to experienced observers. The problem is aggravated when multiple intrageneric species are sympatric in the same river basin and, furthermore, when these species produce fertile hybrids which may backcross and generate a gradient of intermediate phenotypes. This seems to be the case of

Table 7

Loci of the analysed beta-actin gene fragment which are considered to be species-specific tags in the studied Luciobarbus fishes from Iberian rivers; nucleotides or ambiguity codes are indicated for each loci

\begin{tabular}{lcccccc}
\hline \multirow{1}{*}{ Species } & \multicolumn{6}{c}{ Loci with species-specific tags } \\
\cline { 2 - 7 } & 54 & 60 & 117 & 126 & 245 & 279 \\
\hline L. comizo & $\mathrm{R}$ & $\mathrm{C}$ & $\mathrm{R}$ & $\mathrm{Y}$ & $\mathrm{R}$ & $\mathrm{S}$ \\
L. microcephalus & $\mathrm{A}$ & $\mathrm{Y}$ & $\mathrm{R}$ & $\mathrm{T}$ & $\mathrm{G}$ & $\mathrm{K} / \mathrm{D}$ \\
L. sclateri & $\mathrm{R}$ & $\mathrm{C}$ & $\mathrm{G}$ & $\mathrm{Y}$ & $\mathrm{R}$ & $\mathrm{S} / \mathrm{B}$ \\
\hline
\end{tabular}

Table 8

Identification of Luciobarbus sampled in the Guadiana River sub-basins based on phenotypic traits, mtDNA, nDNA, and the combination of mitochondrial and nuclear sequencing

\begin{tabular}{lccccccccc}
\hline \multirow{2}{*}{ Species } & \multicolumn{1}{c}{ Phenotypic } & mtDNA & nDNA & \multicolumn{3}{c}{$\begin{array}{c}\text { Mito- } \\
\text { nuclear }\end{array}$} \\
\cline { 2 - 10 } & \multicolumn{1}{c}{$n$} & {$[\%]$} & $n$ & {$[\%]$} & $n$ & {$[\%]$} & $n$ & {$[\%]$} \\
\hline L. comizo & 42 & 21.7 & 75 & 38.7 & 41 & 21.1 & 35 & 18.0 \\
L. microcephalus & 45 & 23.2 & 33 & 17.0 & 17 & 8.8 & 21 & 10.8 \\
L. sclateri & 74 & 38.1 & 86 & 44.3 & 78 & 40.2 & 59 & 30.4 \\
L. steindachneri & 33 & 17.0 & 0 & 0 & 0 & \\
Hybrids & 0 & & 0 & 58 & 29.9 & 79 & 40.7 \\
Total & 194 & & 194 & 194 & & 194 & \\
\hline
\end{tabular}

Mito-nuclear = mito-nuclear genotype.

Relations between in loco identifications and mito-nuclear genotypes of Luciobarbus sampled in the Guadiana River sub-basins

\begin{tabular}{lccccc}
\hline \multirow{2}{*}{ In loco identification } & \multicolumn{3}{c}{ Mito-nuclear genotype } & Misidentification \\
\cline { 2 - 5 } & L. comizo & L. microcephalus & L. sclateri & Hybrids & \\
\hline L. comizo & $\mathbf{2 6}$ & 0 & 5 & 11 & 38 \\
L. microcephalus & 1 & $\mathbf{8}$ & 17 & 19 & 82 \\
L. sclateri & 6 & 9 & $\mathbf{2 4}$ & 35 & 67.6 \\
L. steindachneri & 2 & 4 & 13 & 14 & 100 \\
Total & 35 & 21 & 59 & 79 & \\
\hline
\end{tabular}



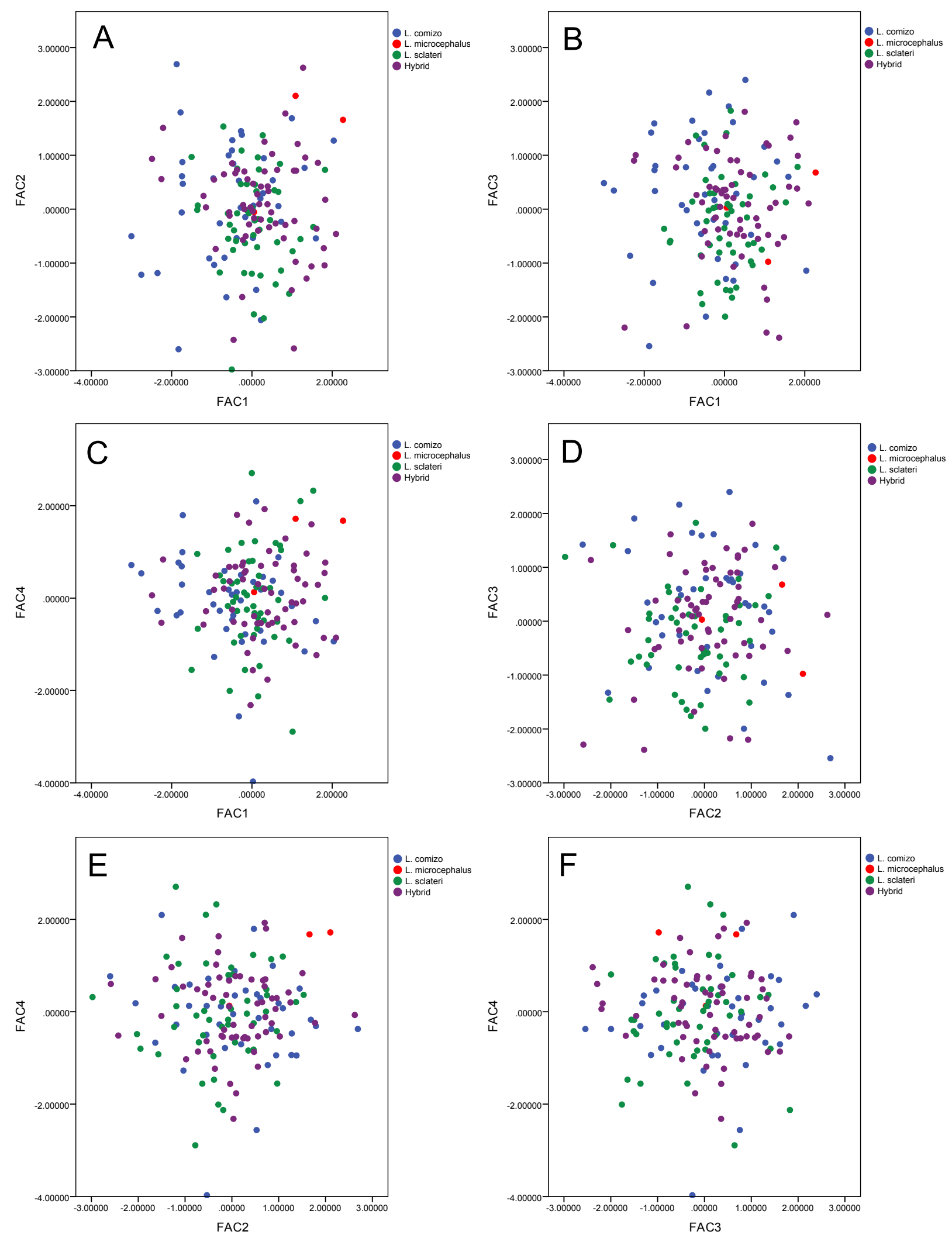

Fig. 4. Scatterplots of the principal component analyses of morphometric variables of Guadiana barbels (species assigned by mito-nuclear analysis): (A) variables FAC1 and FAC2, (B) variables FAC1 and FAC3, (C) variables FAC1 and FAC4, (D) variables FAC2 and FAC3, (E) variables FAC2 and FAC4, (F) variables FAC3 and FAC4; Note: FAC1 through FAC4 are the four new variables created, which explained $83.34 \%$ of the variance 
the Luciobarbus species occurring in the Guadiana basin, considered to configure an extremely interesting model of speciation-with-gene-flow due to weak constraints to hybridization in breeding grounds (Gante et al. 2015). In practical terms, however, this scenario results in the possibility of incorrect species identifications in the field and, consequently, calls into question all the surveys and published data made without molecular validation of the studied individuals. Indeed, our results clearly demonstrate that the in loco species identification based on phenotypic characters, which has been used for the last 30 years, is not reliable to distinguish Luciobarbus species in the lower Guadiana River and, consequently, their relative abundances were overestimated and hybrids, which represented approximately $41 \%$ of the individuals sequenced, were not detected nor considered. Simple morphological analyses using traditional morphometric measures, which could be an alternative to in loco phenotypic identification, are also not reliable since, according to our results, no clear separation between the genetic entities was found in the Guadiana River. More elaborate analyses or the use of geometric morphometrics might improve discrimination and clarify their eventual morphological distinctiveness, however, even if that was the case, the presence of a high percentage of hybrids justifies the use of molecular tools in all the studies conducted with Luciobarbus from the Guadiana River.

The implications of misidentifications might, in some cases, raise high levels of concern since important previously published data on the ecology and biology of the species (e.g., distribution areas, reproductive seasons, local abundance estimates, spawning behaviour, feeding ecology, or growth rates) may be questionable, as well as the conservation statuses assigned. Additionally, the establishment of conservation and management measures is usually made using data on population declines and fragmentation of populations, which may both be compromised by erroneous species identification in the field.

Along with species misidentification, our study also highlights the need to clarify the taxonomy of L. steindachneri since there is no evidence of a significant genetic divergence from the remaining Luciobarbus species that could support its specific status (Gante et al. 2009, 2015, presently reported study). Indeed, our results show that the individuals identified in loco as L. steindachneri were instead interspecific hybrids (with mtDNA of one of the other three Luciobarbus species, indicating that mothers of all the species are involved in hybrid crosses) or showed pure genotypes (mostly of L. sclateri). The validity of this species, described almost 50 years ago based on morphological and meristic data (Almaça 1967), has been questioned (Doadrio 1988, Doadrio et al. 2002). Recently, in line with our findings, Gante et al. (2015) referred to this species as being the local product, in the Guadiana River, of the introgressive hybridization between L. comizo and L. microcephalus or L. sclateri. These authors suggest that L. steindachneri is an ecotype of hybrid origin, with intermediate molecular, morphological, trophic, and ecological characteristics. However, in our view, the maintenance of hybrids as an independent taxonomical entity with a conservation status is questionable and may result in more disadvantages than advantages, so we suggest that L. steindachneri species name should be considered no longer valid. Also regarding taxonomy, the detection of an individual with $L$. microcephalus mtDNA and a mixture of nuclear species-specific tags of $L$. comizo and L. bocagei is worth further investigation. The presence of $L$. bocagei genes outside of the species distribution area may be due to a human introduction. Another hypothesis which cannot be discarded yet is that the specific-tags of L. bocagei at the beta-actin level may be identical to the ones of Luciobarbus guiraonis, a species endemic to the Mediterranean slope of the Iberian Peninsula but which also occurs in some rivers of the upper Guadiana River basin (Doadrio et al. 2011).

Concerning the presence of intrageneric hybrids, previous studies had already reported their occurrence among barbels from Guadiana based on the detection of intermediate phenotypes (Gante et al. 2015). However, our study ads an extra worrisome result by showing that even individuals undoubtedly assigned to a certain species were indeed hybrids when genotyped (cryptic hybrids).

The existence of phenotypically unidentifiable hybrids could also explain the failure to clearly discriminate all the Luciobarbus species occurring in the Guadiana using morphological indices, despite the obtained significant discrimination between $L$. sclateri and L. comizo. These results corroborate the view highlighted by Gante et al. (2015) that the genomes of Iberian sympatric barbels remain porous and allow for gene exchange, despite being sufficiently divergent species. Indeed, previous phylogenies based on two mitochondrial genes calibrated using fossil evidence, showed that the lineage which originated L. microcephalus diverged around $7 \mathrm{Mya}$, and the lineage which originated the other three species was split around 4 Mya, given rise to L. sclateri on one hand, and to L. bocagei and L. comizo on the other hand (these two species were differentiated from each other more recently, around 1.9 Mya) (Gante et al. 2011).

Our study also revealed the occurrence of mito-nuclear discordance in a considerable number of individuals (around 34\% of the total number of individuals sequenced) suggesting the presence of, at least, second-generation hybrids. The presence of cryptic hybrids and mito-nuclear discordances were already reported for a wide variety of animals (Toews and Brelsford 2012), including freshwater fish (Gante et al. 2009, Choleva et al. 2014, Sousa-Santos et al. 2014, Geiger et al. 2016). Mito-nuclear discordance, in particular, likely stem from the loss of a species-specific signal due to lineage sorting and/or non-assortative mating, as already proposed for other cyprinids (Freyhof et al. 2005, Broughton et al. 2011, Sousa-Santos et al. 2014).

Although females of all species of sympatric Luciobarbus (L. comizo, L. sclateri, and L. microcephalus) were involved in interspecific crosses, the prevalence of L. comizo-mtDNA in cryptic hybrids points to an eventual sexually biased direction of hybridization, as already suggested for hybrids between sympatric species of European Barbus (see Lajbner et al. 2009, Meraner et al. 2013, Buonerba et al. 2015). Indeed, as these 
authors suggest, females for the largest species (in our case, L. comizo) are more likely to attract smaller males from other species than vice versa, resulting in a higher percentage of interspecific hybrids carrying the mtDNA of the largest species that would be expected if mating was random. The local relative abundances of sympatric Luciobarbus species might also explain the detected differential contributions of females for interspecific crosses: females of the less common species will most likely produce hybrid progeny since finding a mate among conspecifics will be less probable than mating with congeners or with hybrids. Thus, as discussed by Wirtz (1999) and Rosenthal (2013), the absence of behavioural barriers to interspecific mating may promote hybridization and, furthermore, mate preferences and the scarcity of conspecific mates results in unidirectional hybridization processes. Massive mtDNA unidirectional introgressions attributed to demographical and/or behavioural reasons were already reported for a wide variety of taxa (Wirtz 1999, Ritz et al. 2008, Nevado et al. 2009, Sequeira et al. 2011). Future studies should be designed to allow the establishment of correlations between the type of mtDNA found in hybrid barbels from the Guadiana River and the local relative abundances of each parental species.

Alongside distinct mtDNAs, cryptic hybrids also showed distinct frequencies according to the sub-basin considered, with a tendency to be more frequent in the ones with more dams (Ardila, Chança, and Caia). On the other hand, the higher percentages of concordance between in loco species identifications and mito-nuclear pure genotypes were detected in well preserved and dam free sub-basins (Vascão and Cobres). Thus, we suggest that hybridization may have been potentiated by the loss of river connectivity, which compromises the upstream migration of these potamodromous species and prevents the use of preferred spawning grounds, and by the lower availability of adequate habitats in more artificialized river systems. Positive correlations between damming and the occurrence of hybrids were already reported (Hasselman et al. 2014). This will undoubtedly lead to genetic homogenization, culminating in a loss of biodiversity.

The proven inadequate phenotypic diagnostic characters, the occurrence of cryptic hybrids in such an expressive percentage and the suggestion to fail to consider L. steindachneri as an independent taxonomical entity (and instead consider these individuals as interspecific hybrids) highlight the need to a careful review of the previously published data on biological and ecological features of Luciobarbus species. Furthermore, on-going conservation measures for threatened barbel populations should be reviewed in view of this hybrid puzzle scenario.

Several cases of hybridization between barbels were also reported elsewhere in Europe, based on the occurrence of morphologically intermediate hybrids and, less frequently, on inconsistencies between phenotypes and mitochondrial genotypes (reviewed by Geiger et al. 2016). Thus, the herein proposed use of a combination of mitochondrial and nuclear markers as a reliable method to non-erroneously identify barbels in the Guadiana River should become widely used in those river systems where different intrageneric sympatric species with soft mechanisms of reproductive isolation that might potentially interbreed. Reliable taxonomical assignments are crucial for species preservation since successful conservation plans need to consider the genetic integrity of their conservation units. We thus suggest that mito-nuclear sequencing becomes a standard practice to correctly identify fish where sympatric hybridizing species occur.

\section{ACKNOWLEDGMENTS}

We thank G. Lemos, C. Carrapato, and C. Cardoso for their help during sampling. Permits for field work were given by the National Institute for the Conservation of Nature and Forests (ICNF), Portugal. This study was financed by the European Fund for Economic and Regional Development (FEDER) through the Program Operational Factors of Competitiveness (COMPETE) and National Funds through the FCT - Portuguese Foundation of Science and Technology, under the Pluriannual Program UI\&D 331/94; the strategic project UID/MAR/04292/2013 granted to MARE and UID/AGR/00239/2013 granted to CEF-ISA; the project PTDC/AAC-CLI/103110/2008; the projects PTDC/ AAC-CLI/103110/2008 and Pest-OE/AGR/UI0239/2014; and the grants awarded to C. Sousa-Santos (SFRH/ BPD/29774/2006 and MARE-ISPA/BPD/001/2015), C. Lima (MARE-ISPA/BI/004/2015), and P. Branco (SFRH/ BPD/94686/2013). José Maria Santos was funded by a postdoctoral grant (MARS/BI/2/2014) from the MARS project (http://www.mars-project.eu/) and is presently recipient of a FCT researcher contract (IF/00020/2015).

\section{REFERENCES}

Aboim M.A., Mavárez J., Bernatchez L., Coelho M.M. 2010. Introgressive hybridization between two Iberian endemic cyprinid fish: A comparison between two independent hybrid zones. Journal of Evolutionary Biology 23 (4): 817-828. DOI: 10.1111/j.14209101.2010.01953.x

Almaça C. 1967. Estudo das populações portuguesas do gén. Barbus Cuvier, 1817 (Pisces, Cyprinidae). [Study of the Portuguese populations of the genus Barbus Cuvier, 1817 (Pisces, Cyprinidae).] Revista da Faculdade de Ciências de Lisboa $2^{\mathrm{a}}$ série, C 14 (2): 151-400. [In Portuguese.]

Almaça C. 1972. Sur la systématique des barbeaux (genre et sous-genre Barbus) de la Penínsule Ibérique et de 1'Afrique du Nord. Arquivos do Museu Bocage $2^{\mathrm{a}}$ série 3 (10): 319-346.

Almaça C., Bănărescu P.M. 2003. Barbus comizo Steindachner, 1865. Pp. 173-180. In: Bănărescu P.M., Bogutskaya N.G. (eds.) The freshwater fishes of Europe. Aula-Verlag, Wiesbaden, Germany.

Almodóvar A., Nicola G.G., Elvira B. 2008. Natural hybridization of Barbus bocagei $\times$ Barbus comizo (Cyprinidae) in Tagus River basin, central Spain. Cybium 32 (2): 99-102. 
Armbruster J.W. 2012. Standardized measurements, landmarks, and meristic counts for cypriniform fishes. Zootaxa 3586: 8-16.

Arnold M.L. 2006. Evolution through genetic exchange. Oxford University Press, Oxford, UK.

Barton N.H. 2001. The role of hybridization in evolution. Molecular Ecology 10 (3): 551-568. DOI: 10.1046/j.1365-294x.2001.01216.x

Barton N.H., Hewitt G.M. 1985. Analysis of hybrid zones. Annual Review of Ecology and Systematics 16: 113-148. DOI: 10.1146/annurev.es.16.110185. 000553

Bhangale T.R., Stephens M., Nickerson D.A. 2006. Automating resequencing-based detection of insertion-deletion polymorphisms. Nature Genetics 38 (12): 1457-1462. DOI: 10.1038/ng1925

Bianco P.G. 1982. Hybridization between Alburnus albidus (C.) and Leuciscus cephalus cabeda R. in Italy. Journal of Fish Biology 21 (5): 593-603. DOI: 10.1111/j.1095-8649.1982.tb02862.x

Bianco P.G., Ketmaier V. 2001. Anthropogenic changes in the freshwater fish fauna of Italy, with reference to the central region and Barbus graellsii, a newly established alien species of Iberian origin. Journal of Fish Biology 59 (Suppl. A): 190-208. DOI: 10.1111/ j.1095-8649.2001.tb01386.x

Bohling J.H. 2016. Strategies to address the conservation threats posed by hybridization and genetic introgression. Biological Conservation 203: 321-327. DOI: 10.1016/j.biocon.2016.10.011

Brito R.M., Briolay J., Galtier N., Bouvet Y., Coelho M.M. 1997. Phylogenetic relationships within the genus Leuciscus (Pisces, Cyprinidae) in Portuguese fresh waters, based on mitochondrial DNA cytochrome $b$ sequences. Molecular Phylogenetics and Evolution 8 (3): 435-442. DOI: 10.1006/mpev.1997.0429

Broughton R.E., Vedala K.C., Crowl T.M., Ritterhouse L.L. 2011. Current and historical hybridization with differential introgression among three species of cyprinid fishes (genus Cyprinella). Genetica 139 (5): 699-707. DOI: 10.1007/s10709-011-9578-9

Buonerba L., Zaccara S., Delmastro G.B., Lorenzoni M., Salzburger W., Gante H.F. 2015. Intrinsic and extrinsic factors act at different spatial and temporal scales to shape population structure, distribution and speciation in Italian Barbus (Osteichthyes: Cyprinidae). Molecular Phylogenetics and Evolution 89: 115-129. DOI: 10.1016/j.ympev.2015.03.024

Cabral M.J., Almeida J., Almeida P.R., Dellinger T., Ferrand de Almeida N., Oliveira M.E., Palmeirim J.M., Queirós A.I., Rogado L., Santos-Reis M. (eds.) 2005. Livro vermelho dos vertebrados de Portugal. [Red book of the vertebrates of Portugal.] Instituto da Conservação da Natureza, Lisboa, Portugal. [In Portuguese.]

Callejas C., Ochando M.D. 2002. Phylogenetic relationships among Spanish Barbus species (Pisces, Cyprinidae) shown by RAPD markers. Heredity 89 (1): 36-43. DOI: 10.1038/sj.hdy.6800091
Chen K., McLellan M.D., Ding L., Wendl M.C., Kasai Y., Wilson R.K., Mardis E.R. 2007. PolyScan: An automatic indel and SNP detection approach to the analysis of human resequencing data. Genome Research 17 (5): 659-666. DOI: 10.1101/gr.6151507

Choleva L., Musilova Z., Kohoutova-Sediva A., Paces J., Rab P., Janko K. 2014. Distinguishing between incomplete lineage sorting and genomic introgressions: Complete fixation of allospecific mitochondrial DNA in a sexually reproducing fish (Cobitis; Teleostei), despite clonal reproduction of hybrids. PLoS ONE 9 (6): e80641. DOI: 10.1371/journal.pone.0080641

Congiu L., Dupanloup I., Patarnello T., Fontana F., Rossi R., Arlati G., Zane L. 2001. Identification of interspecific hybrids by amplified fragment length polymorphism: The case of sturgeon. Molecular Ecology 10 (9): 2355-2359. DOI: 10.1046/j.09621083.2001.01368.x

Costedoat C., Pech N., Chappaz R., Gilles A. 2007. Novelties in hybrid zones: Crossroads between population genomic and ecological approaches. PLoS ONE 2 (4): e357. DOI: 10.1371/journal.pone.0000357

Dmitriev D.A., Rakitov R.A. 2008. Decoding of superimposed traces produced by direct sequencing of heterozygous indels. PLoS Computational Biology 4 (7): e1000113. DOI: 10.1371/journal.pcbi.1000113

Doadrio I. (ed.) 2001. Atlas y libro rojo de los peces continentales de España. Dirección General de Conservación de la Naturaléza, Museo Nacional de Ciencias Naturales, Madrid, Spain.

Doadrio I. 1988. Sobre la taxonomia de Barbus comiza Steindachner, 1865 (Ostariophysi: Cyprinidae). Doñana Acta Vertebrata 15 (1): 19-28.

Doadrio I., Carmona J.A., Machordom A. 2002. Haplotype diversity and phylogenetic relationships among the Iberian Barbels (Barbus, Cyprinidae) reveal two evolutionary lineages. Journal of Heredity 93 (2): 140-147. DOI: 10.1093/jhered/93.2.140

Doadrio I., Elvira B., Bernat Y. 1991. Peces continentales españoles. Inventario y clasificación de zonas fluviales. Instituto para la Conservación de la Naturaleza-Consejo Superior de Investigaciones Científicas (ICONA-CSIC), Madrid, Spain.

Doadrio I., Perea S., Garzón-Heydt P., González J.L. 2011. Ictiofauna continental española. Bases para su seguimiento. DG Medio Natural y Política Forestal, MARM, Madrid, Spain.

Encina L., Granado-Lorencio C. 1990. Morfoecología trófica en el género Barbus (Pisces, Cyprinidae). Limnetica 6 (1): 35-46.

Freyhof J., Lieckfeldt D., Pitra C., Ludwig A. 2005. Molecules and morphology: Evidence for introgression of mitochondrial DNA in Dalmatian cyprinids. Molecular Phylogenetics and Evolution 37 (2): 347-354. DOI: 10.1016/j.ympev.2005.07.018

Froese R., Pauly D. (eds.) 2017. FishBase. [Version 01/2017] www.fishbase.org

Gante H.F., Alves M.J., Dowling T.E. 2011. Paralogspecific primers for the amplification of nuclear loci 
in tetraploid barbels (Barbus: Cypriniformes). Journal of Heredity 102 (5): 617-621. DOI: 10.1093/jhered/ esr059

Gante H.F., Doadrio I., Alves M.J., Dowling T.E. 2015. Semi-permeable species boundaries in Iberian barbels (Barbus and Luciobarbus, Cyprinidae). BMC Evolutionary Biology 15: e111. DOI: 10.1186/s12862015-0392-3

Gante H.F., Micael J., Oliva-Paterna F.J., Doadrio I., Dowling T.E., Alves M.J. 2009. Diversification within glacial refugia: Tempo and mode of evolution of the polytypic fish Barbus sclateri. Molecular Ecology 18 (15): 3240-55. DOI: 10.1111/j.1365294X.2009.04264.X

Geiger M.F., Schreiner C., Delmastro G.B., Herder F. 2016. Combining geometric morphometrics with molecular genetics to investigate a putative hybrid complex: A case study with barbels Barbus spp. (Teleostei: Cyprinidae). Journal of Fish Biology 88 (3): 1038-1055. DOI: $10.1111 / \mathrm{jfb} .12871$

Gerlach G., Atema J., Raupach M.J., Deister F., Müller A., Kingsford M.J. 2016. Cryptic species of cardinalfish with evidence for old and new divergence. Coral Reefs 35 (2): 437. DOI: 10.1007/s00338-0151395-7

Godinho F.N., Ferreira M.T., Cortes R. 1997. Composition and spatial organization of fish assemblages in the lower Guadiana basin, southern Iberia. Ecology of Freshwater Fish 6 (3): 134-143. DOI: $10.1111 /$ j.1600-0633.1997.tb00155.X

Hammer Ø., Harper D.A.T., Ryan P.D. 2009. PAST PAlaeontological STatistics, ver. 1.89. Palaeontologia Electronica 4 (1): 9pp.

Hänfling B., Bolton P., Harley M., Carvalho G.R. 2005. A molecular approach to detect hybridisation between crucian carp (Carassius carassius) and nonindigenous carp species (Carassius spp. and Cyprinus carpio). Freshwater Biology 50 (3): 403-417. DOI: 10.1111/j.1365-2427.2004.01330.x

Hasselman D.J., Argo E.E., McBride M.C., Bentzen P., Schultz T.F., Perez-Umphrey A.A., Palkovacs E.P. 2014. Human disturbance causes the formation of a hybrid swarm between two naturally sympatric fish species. Molecular Ecology 23 (5): 1137-1152. DOI: 10.1111/mec.12674

Kottelat M. 1997. European freshwater fishes. An heuristic checklist of the freshwater fishes of Europe (exclusive of former USSR), with an introduction for non-systematists on nomenclature and conservation. Biologia (Bratislava), Section Zoology 52 (Suppl. 5): 1-271.

Kottelat M., Freyhof J. 2007. Handbook of European freshwater fishes. Kottelat, Cornol, Switzerland and Freyhof, Berlin, Germany.

Kuparinen A., Vinni M., Teacher A.G.F., Kähkönen K., Merilä J. 2014. Mechanism of hybridization between bream Abramis brama and roach Rutilus rutilus in their native range. Journal of Fish Biology 84 (1): 237-242. DOI: $10.1111 / \mathrm{jfb} .12272$
Lajbner Z., Šlechtová V., Šlechta V., Švátora M., Berrebi P., Kotlík P. 2009. Rare and asymmetrical hybridization of the endemic Barbus carpathicus with its widespread congener Barbus barbus. Journal of Fish Biology 74 (2): 418-436. DOI: 10.1111/j.10958649.2008.02098.x

Lobón-Cervia J., Fernández-Delgado C. 1984. On the biology of the barbel (Barbus bocagei) in the Jarama River. Folia Zoologica 33 (4): 371-384.

Machordom A., Berrebi P., Doadrio I. 1990. Spanish barbel hybridization detected using enzymatic markers: Barbus meridionalis Risso $\times$ Barbus haasi Mertens (Osteichthyes, Cyprinidae). Aquatic Living Resources 3 (4): 295-303. DOI: 10.1051/alr:1990030

Mallet J. 2005. Hybridization as an invasion of the genome. Trends in Ecology and Evolution 20 (5): 229-237. DOI: 10.1016/j.tree.2005.02.010

Matondo B.N., Ovidio M., Poncin P., Philippart J.-C. 2010. Eco-ethological characteristics of two natural hybrids of Abramis brama (L.) from the River Meuse basin. Environmental Biotechnology 6 (2): 42-52.

Meraner A., Venturi A., Ficetola G.F., Rossi S., Candiotto A., Gandolfi A. 2013. Massive invasion of exotic Barbus barbus and introgressive hybridization with endemic Barbus plebejus in northern Italy: Where, how and why? Molecular Ecology 22 (21): 5295-5312. DOI: 10.1111/mec. 12470

Morán-López R., Pérez-Bote J.L., Da Silva Rubio E., Corbacho Amado C. 2005. Summer habitat relationships of barbels in south-west Spain. Journal of Fish Biology 67 (1): 66-82. DOI: 10.1111/j.00221112.2005.00711.x

Nevado B., Koblmüller S., Sturmbauer C., Snoeks J., Usano-Alemany J., Verheyen E. 2009. Complete mitochondrial DNA replacement in a Lake Tanganyika cichlid fish. Molecular Ecology 18 (20): 4240-4255. DOI: 10.1111/j.1365-294X.2009.04348.x

Paterson I.D., Mangan R., Downie D.A., Coetzee J.A., Hill M.P., Burke A.M., Downey P.O., Henry T.J., Compton S.G. 2016. Two in one: Cryptic species discovered in biological control agent populations using molecular data and crossbreeding experiments. Ecology and Evolution 6 (17): 6139-6150. DOI: 10.1002/ece3.2297

Pereira C., Neto A., Collares-Pereira M.J. 2009. Cytogenetic survey of species of two distinct genera of Iberian nases (Cyprinidae, Leuciscinae) that hybridize extensively in nature. I. evidence of a similar and conserved chromosome pattern with some few speciesspecific markers at macro-structural leve. Genetica 137 (3): 285-291. DOI: 10.1007/s 10709-009-9379-6

Pereira C.S.A., Aboim M.A., Ráb P., Collares-Pereira M.J. 2014. Introgressive hybridization as a promoter of genome reshuffling in natural homoploid fish hybrids (Cyprinidae, Leuciscinae). Heredity 112 (3): 343-350. DOI: 10.1038/hdy.2013.110

Pires A.M., Cowx I.G., Coelho M.M. 2001. Diet and growth of the two sympatric Iberian barbel, Barbus steindachneri and Barbus microcephalus, in the 
middle reaches of the Guadiana basin (Portugal). Folia Zoologica 50 (4): 291-304.

Ráb P., Collares-Pereira M.J. 1995. Chromosomes of European cyprinid fishes (Cyprinidae, Cypriniformes): A review. Folia Zoologica 44 (3): 193-214.

Ritz M.S., Millar C., Miller G.D., Phillips R.A., Ryan P., Sternkopf V., Liebers-Helbig D., Peter H.-U. 2008. Phylogeography of the southern skua complexrapid colonization of the Southern Hemisphere during a glacial period and reticulate evolution. Molecular Phylogenetics and Evolution 49 (1): 292-303. DOI: 10.1016/j.ympev.2008.07.014

Robalo J.I., Almada V.C., Levy A., Doadrio I. 2007. Re-examination and phylogeny of the genus Chondrostoma based on mitochondrial and nuclear data and the definition of 5 new genera. Molecular Phylogenetics and Evolution 42 (2): 362-372. DOI: 10.1016/j.ympev.2006.07.003

Rosenthal G.G. 2013. Individual mating decisions and hybridization. Journal of Evolutionary Biology 26 (2): 252-255. DOI: 10.1111/jeb.12004

Scheet P., Stephens M. 2006. A fast and flexible statistical model for large-scale population genotype data: Applications to inferring missing genotypes and haplotypic phase. American Journal of Human Genetics 78 (4): 629-644. DOI: 10.1086/502802

Schindelin J., Arganda-Carreras I., Frise E., Kaynig V., Longair M., Pietzsch T., Preibisch S., Rueden C., Saalfeld S., Schmid B., Tinevez J.-Y., White D.J., Hartenstein V., Eliceiri K., Tomancak P., Cardona A. 2012. Fiji: An open-source platform for biologicalimage analysis. Nature Methods 9 (7): 676-682. DOI: 10.1038/nmeth.2019

Schmidt T.R., Gold J.R. 1993. Complete sequence of the mitochondrial cytochrome $b$ gene in the cherryfin shiner, Lythrurus roseipinnis (Teleostei: Cyprinidae). Copeia 1993 (3): 880-883. DOI: 10.2307/1447258

Schumer M., Rosenthal G.G., Andolfatto P. 2014. How common is homoploid hybrid speciation? Evolution 68 (6): 1553-1560. DOI: 10.1111/evo.12399

Scribner K.T., Page K.S., Bartron M.L. 2000. Hybridization in freshwater fishes: A review of case studies and cytonuclear methods of biological inference. Reviews in Fish Biology and Fisheries 10 (3): 293-323. DOI: 10.1023/A:1016642723238

Sequeira F., Sodré D., Ferrand N., Bernardi J.A.R., Sampaio I., Schneider H., Vallinoto M. 2011. Hybridization and massive mtDNA unidirectional introgression between the closely related Neotropical toads Rhinella marina and $R$. schneideri inferred from mtDNA and nuclear markers. BMC Evolutionary Biology 11: 264. DOI: 10.1186/1471-2148-11-264

Sousa-Santos C., Gante H.F., Robalo J., Proença Cunha P., Martins A., Arruda M., Alves M.J., Almada V. 2014. Evolutionary history and population genetics of a cyprinid fish (Iberochondrostoma olisiponensis) endangered by introgression from a more abundant relative. Conservation Genetics 15 (3): 665-677. DOI: 10.1007/s10592-014-0568-1

Sousa-Santos C., Robalo J.I., Collares-Pereira M.-J., Almada V.C. 2005. Heterozygous indels as useful tools in the reconstruction of DNA sequences and in the assessment of ploidy level and genomic constitution of hybrid organisms. DNA Sequence 16 (6): 462467. DOI: 10.1080/10425170500356065

Sousa-Santos C., Robalo J.I., Pereira A.M., Branco P., Santos J.M., Ferreira M.T., Sousa M., Doadrio I. 2016. Broad-scale sampling of primary freshwater fish populations reveals the role of intrinsic traits, inter-basin connectivity, drainage area and latitude on shaping contemporary patterns of genetic diversity. PeerJ 4: e1694. DOI: 10.7717/peerj.1694

Stephens M., Donnelly P. 2003. A comparison of Bayesian methods for haplotype reconstruction from population genotype data. American Journal of Human Genetics 73 (5): 1162-1169. DOI: 10.1086/379378

Stephens M., Smith N., Donnelly P. 2001. A new statistical method for haplotype reconstruction from population data. American Journal of Human Genetics 68 (4): 978-989. DOI: 10.1086/319501

Taylor E.B., Boughman J.W., Groenenboom M., Sniatynski M., Schluter D., Gow J.L. 2006. Speciation in reverse: Morphological and genetic evidence of the collapse of a three-spined stickleback (Gasterosteus aculeatus) species pair. Molecular Ecology 15 (2): 343-355. DOI: 10.1111/j.1365294X.2005.02794.x

Toews D.P.L., Brelsford A. 2012. The biogeography of mitochondrial and nuclear discordance in animals. Molecular Ecology 21 (16): 3907-3930. DOI: 10.1111/j.1365-294X.2012.05664.x

Ünver B., Erk'akan F. 2005. A natural hybrid of Leuciscus cephalus (L.) and Chalcalburnus chalcoides (Güldenstädt) (Osteichthyes-Cyprinidae) from Lake Tödürge (Sivas, Turkey). Journal of Fish Biology 66 (4): 899-910. DOI: 10.1111/j.0022-1112.2005.00610.x

Wirtz P. 1999. Mother species-father species: Unidirectional hybridization in animals with female choice. Animal Behaviour 58 (1): 1-12. DOI: 10.1006/ anbe.1999.1144

Witkowski A., Kotusz J., Wawer K., Stefaniak J., Popiołek M., Błachuta J. 2015. A natural hybrid of Leuciscus leuciscus (L.) and Alburnus alburnus (L.) (Osteichthyes: Cyprinidae) from the Bystrzyca River (Poland). Annales Zoologici 65 (2): 287-293. DOI: 10 .3161/00034541ANZ2015.65.2.010

Zamora L., Almeida D (eds.) 2015. Carta Piscícola Española. Sociedad Ibérica de Ictiología (SIBIC) [version 01/2015] http://www.cartapiscicola.es/\#/ home 
